\title{
Teoria de Nielsen de raízes para aplicações equivariantes
}

\author{
Hildebrane Augusto dos Santos
}

\author{
TESE APRESENTADA \\ $\mathrm{AO}$ \\ INSTITUTO DE MATEMÁTICA E ESTATÍSTICA \\ DA \\ UNIVERSIDADE DE SÃO PAULO \\ PARA \\ OBTENÇÃO DO TÍTULO \\ $\mathrm{DE}$ \\ DOUTOR EM CIÊNCIAS
}

Área de Concentração: Matemática

Orientador: Prof. Dr. Peter N-S Wong

Durante a elaboração deste trabalho, a autora recebeu apoio financeiro do CNPq e da CAPES

- São Paulo, fevereiro de 2009 - 


\title{
Teoria de Nielsen de raízes para aplicações equivariantes
}

\author{
Este exemplar corresponde à redação final \\ da tese de doutorado devidamente \\ corrigida e defendida por \\ Hildebrane Augusto dos Santos \\ e aprovada pela comissão julgadora.
}

São Paulo, 18 de março de 2009.

\section{Comissão Julgadora:}

Prof. Dr. Peter N-S Wong (Orientador) - Bates College

Profa. Dra. Fernanda Soares P. Cardona - IME-USP

Profa. Dra. Alice Kimie Miwa Libardi - UNESP-Rio Claro

Prof. Dr. Daniel Vendrúscolo - DM-UFCar

Prof.Dr. Oziride Manzoli Neto - ICMC-USP-São Carlos 


\section{Agradecimentos}

À minha família, pelo amor e pela torcida.

Ao meu orientador Prof. Dr. Peter Wong sou imensamente grata pela dedicação, incentivo e apoio constantes na orientação deste trabalho, sem os quais, obviamente, não teria condições de concluí-lo. Foi uma grande satisfação poder contar com sua longa experiência.

À Profa. Dra. Fernanda Cardona meus agradecimentos por sua permanente solicitude em todas as fases do projeto, pela imensa paciência e por brindar-me com uma colaboração importante para este trabalho, dosando as críticas com comentários de incentivo.

À Profa. Dra. Lucília Borsari e à Profa. Dra. Natalia Viana Bedoya agradeço as ricas sugestões a esse trabalho.

Aos Professores do Departamento de Matemática do IME, em especial, ao Prof. Dr. Daciberg Lima Gonçalves e ao Prof. Dr. Jorge Hiratuka.

Ao Leonardo, pela amizade sempre sincera.

À Fernanda, amiga de todas as horas, pela solidariedade e amizade compartilhadas todo esse tempo.

À Andréia, Patrícia e Fernandinha pelo companheirismo e encorajamento nas horas mais difíceis.

Aos meus colegas do grupo de Topologia Algébrica, em particular, aos alunos Paulo, Anderson, Toninho e Gustavo pelo convívio e pela rica troca de idéias matemáticas.

À Susan e a Laura, pelo carinho e atenção. 
À Georgette Dumais, à Profa. Dra. Maria Ortiz e ao Prof. Dr. Eduardo Muslip do Bates College pela calorosa recepção em Lewiston, pela amizade e apoio constantes. Meu muito obrigada por todos os momentos compartilhados.

À todas as pessoas que colaboraram, direta ou indiretamente, para a realização deste trabalho.

À CAPES e ao CNPq pelo apoio financeiro. 


\section{Resumo}

Este trabalho consiste de duas partes. Na primeira, desenvolvemos uma teoria de Nielsen equivariante para raízes de $G$-aplicações. Consideramos aplicações $f: X \rightarrow Y$ equivariantes entre G-espaços topológicos Hausdorff, conexos, normais, localmente conexos por caminhos e semilocalmente simplesmente conexos, onde $G$ é um grupo topológico. Na segunda parte, estudamos a questão da realização do G-número de Nielsen de raízes quando este é zero.

Palavras-chave: Número de Nielsen de raízes, número de Reidemeister de raízes, $G$-número de Nielsen de raízes, $G$-número de Reidemeister de raízes, revestimento de Hopf. 


\section{Abstract}

This work consists of two parts. In the first one, we develop an equivariant Nielsen root theory for $G$-maps. We consider equivariant maps $f: X \rightarrow Y$ between Hausdorff, connected, normal, locally path connected and semilocally simply connected $G$-spaces, where $G$ is a topological group. In the second part, we study the question of the realization of $G$-Nielsen root number when it is zero.

Keywords: Nielsen root number, Reidemeister root number, G-Nielsen root number, $G$-Reidemeister root number, Hopf covering map. 


\section{Conteúdo}

Agradecimentos $\quad$ i

Resumo $\quad$ iii

Abstract $\quad$ iv

$\begin{array}{ll}\text { Introdução } & 1\end{array}$

1 Preliminares $\quad 4$

1.1 Ação de Grupos . . . . . . . . . . . . . . . . . . . 4

$1.2 \quad G$-Espaço Induzido . . . . . . . . . . . . . . 6

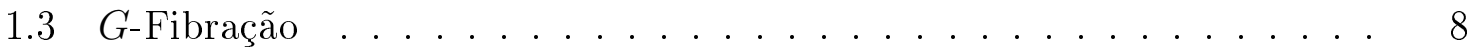

1.4 Teoria de Nielsen de Raízes . . . . . . . . . . . . . . 8

2 Teoria de Nielsen Equivariante $\quad 13$

$2.1 \quad$ G-Classes de Nielsen de raízes . . . . . . . . . . . . . . . . 14

2.2 G-Número de Nielsen de Raízes . . . . . . . . . . . . . . 16

2.3 Levantamento da $G$-ação em $Y \ldots \ldots$. . . . . . . . . . 19

2.4 Relação entre classes e $G$-classes de raízes . . . . . . . . . . . 25

2.5 O G-número de Reidemeister de raízes . . . . . . . . . . . . 27

3 Propriedade Wecken Equivariante 33

$3.1 \quad G$-ANR e $G$-ANE . . . . . . . . . . . . . . 34 
3.2 Propriedade Wecken Equivariante . . . . . . . . . . . 35

$\begin{array}{ll}\text { Considerações finais } & 42\end{array}$

Referências Bibliográficas $\quad 44$ 


\section{Introdução}

Seja $f: X \rightarrow Y$ uma aplicação contínua entre $n$-variedades diferenciáveis fechadas orientadas e seja $a \in Y$ um valor regular. O grau topológico de Brouwer, degf, é uma contagem algébrica do conjunto $f^{-1}(a)$. Em 1930, H. Hopf generalizou este grau para aplicações entre variedades que não são necessariamente orientáveis, utilizando a teoria de Nielsen de raízes como base para a sua teoria do grau (vide [H]). Seguindo o trabalho de J. Nielsen na teoria de ponto-fixo, Hopf introduziu uma relação de equivalência no conjunto $f^{-1}(a)$ e definiu um número de Nielsen de raízes (de $f$ em $a$ ), denotado por, $N(f, a)$, que é um limitante inferior para a cardinalidade de $f^{-1}(a)$, e constitui um invariante homotópico. Hopf mostrou que se $\operatorname{deg} f=0$ então $N(f, a)=0$. Embora seja possível definir $N(f, a)$, mesmo quando $X$ e $Y$ não são variedades topológicas, é usualmente muito difícil (quando possível) calcular este número com tal generalidade.

F. Wecken, no início de 1940, mostrou que para auto-aplicacões de uma variedade compacta triangulável de dimensão pelo menos três, o número de Nielsen de ponto-fixo, o qual é um limitante inferior para o número de pontos-fixos, pode ser realizado na classe de homotopia da aplicação dada, isto é, existe uma aplicação homotópica à aplicação dada cujo número de pontos-fixos é exatamente o número de Nielsen (de ponto-fixo). Em particular, quando o número de Nielsen de ponto-fixo é zero a aplicação dada é deformável para ser livre de ponto-fixo. Em 1955, H. Schirmer [S] provou um teorema tipo Wecken para coincidências de duas aplicações entre variedades fechadas orientáveis e trianguláveis de mesma dimensão. Quando o contra-domínio é uma variedade, a teoria de Nielsen de raízes é um caso especial da teoria de Nielsen de coincidência, 
onde uma das aplicações é a aplicação constante. Logo, se $f: X \rightarrow Y$ é uma aplicação entre $n$-variedades fechadas orientáveis e trianguláveis então se $N(f, a)=0$, existe uma aplicação $h: X \rightarrow Y$ homotópica a $f$ tal que $h^{-1}(a)=\emptyset$.

Influenciado pela teoria de ponto-fixo de Nielsen, R. Brooks [B], em 1967, estendeu a teoria de Nielsen de raízes para espaços mais gerais e definiu a noção de essencialidade de uma classe de raízes sem usar índice. Uma classe de raízes é inessencial se ela "desaparece" por alguma homotopia de $f$ e é essencial caso contrário. O número de Nielsen (geométrico) de raízes é definido como o número de classes de raízes essenciais. A teoria de Nielsen de raízes ganhou muita atenção nos últimos anos (p. ex. [BS]), enquanto que a propriedade Wecken para raízes não tem sido muito estudada em situações onde os espaços não são variedades ou são variedades de dimensões diferentes. Um dos primeiros artigos nessa direção foi $[\mathrm{GW}]$, onde D. Gonçalves e P. Wong provaram, em 2005, que se $X$ é um espaço topológico compacto e conexo por caminhos e $Y$ é uma $n$-variedade topológica (conexa por caminhos mas não necessariamente compacta), então o número de Nielsen de raízes ser nulo é suficiente para garantir a existência de uma aplicação livre de raízes.

Em [W4], P. Wong definiu o $G$-número de Nielsen de raízes, denotado por $N_{G}(f, a)=$ 0, para $G$-aplicações $f: X \rightarrow Y, G$ é um grupo de Lie compacto e $X$ e $Y$ são $G$-ENRs compactos. Um dos objetivos deste trabalho é provar uma versão equivariante de [GW], ou seja, sob que condições $N_{G}(f, a)=0$ garante a existência de uma $G$-aplicação livre de raízes que é $G$-homotópica a $f$ ?

Outro problema em qualquer tipo de teoria de Nielsen é o cálculo do número de Nielsen. Para o cálculo do número de Nielsen de raíz, a ferramenta mais importante é o número de Reidemeister de raízes $R(f, a)$, que é, por definição, a cardinalidade do conjunto $\pi_{1}(Y, f(x)) / f_{\sharp} \pi_{1}(X, x)$. Este número, definido por R. Brooks [B1], coincide com a cardinalidade de $\eta^{-1}(y)$, para qualquer $y \in Y$, onde $\eta: \hat{Y} \rightarrow Y$ é o revestimento de Hopf para $f$ (vide [B3]).

O G-número de Reidemeister de raízes, $R_{G}(f, a)$, também foi definido por P. Wong 
em 1999 [W4], utilizando os espaços de revestimento universais de $X$ e de $Y$. Usando a principal ferramenta deste trabalho, que é o revestimento de Hopf para $f$, daremos uma outra definição de $R_{G}(f, a)$ e mostraremos sua relação com os números $N_{G}(f, a)$ e $R(f, a)$.

Este trabalho está organizado da seguinte forma: no capítulo 1, são apresentados alguns resultados básicos sobre grupos de transformação e será feita uma exposição dos fatos essenciais sobre teoria de Nielsen de raízes.

No capítulo 2, desenvolvemos a teoria de Nielsen equivariante para raízes de $G$ aplicações $f: X \rightarrow Y$. Definimos uma $G$-ação no espaço de revestimento de Hopf de $Y$. Em seguida, damos uma interpretação de essencialidade para $G$-classes de raízes, a qual é uma versão equivariante do Teorema (3.4) de [B3]. Definimos o G-número de Reidemeister de raíz, usando revestimento de Hopf, o qual mostraremos ser um limitante superior para o $G$-número de Nielsen de raízes.

No capítulo 3 são apresentados os resultados obtidos na realização do número de Nielsen equivariante, quando este é zero. 


\section{Capítulo 1}

\section{Preliminares}

Neste capítulo serão fixadas terminologia e notações, e serão apresentados, sem demonstração, alguns resultados fundamentais de ação de grupos e da teoria de Nielsen de raízes para referência futura. Para as demonstrações e uma discussão em detalhes dos resultados, referimos o leitor aos livros [Br], [B3], [Ka], [K] e [tD].

Todo espaço topológico será assumido como sendo normal, Hausdorff, conexo, localmente conexo por caminhos e semilocalmente simplesmente conexo, todas as aplicações são contínuas e as variedades topológicas (espaços paracompactos localmente euclidianos) serão consideradas sem bordo. O termo G-espaço significa $G$-espaço topológico à esquerda, isto é, um espaço $X$ munido de uma ação (contínua) $G \times X \rightarrow X,(g, x) \mapsto g x$.

\subsection{Ação de Grupos}

Nesta seção, introduziremos a terminologia e alguns resultados básicos sobre ação de grupos. Sejam $G$ um grupo topológico e $X$ um $G$-espaço. A órbita de um elemento $x \in X$ é o conjunto $G(x)=\{g x \in X \mid g \in G\}$. O conjunto $G_{x}=\{g \in G \mid g x=x\}$ é um subgrupo de $G$, chamado o grupo de isotropia (ou estabilizador) do ponto $x \in X$. Dizemos que a $G$-ação em $X$ é

(1) trivial se $G_{x}=G$ para todo $x \in X$. 
(2) livre se $G_{x}=\{e\}$ para todo $x \in X$, onde $e$ denota o elemento identidade de $G$.

(3) semi-livre se $G_{x}=G$ ou $G_{x}=\{e\}$ para todo $x \in X$.

(4) transitiva se existe um única órbita, a saber, o espaço $X$.

Dados dois $G$-espaços $X$ e $Y$, uma aplicação $f: X \rightarrow Y$ é chamada G-aplicação ou aplicação equivariante se $f(g x)=g f(x)$, para qualquer $g \in G$ e $x \in X$. Convém observarmos que será usada a mesma notação para as $G$-ações em $X$ e em $Y$.

Um subconjunto $A$ de um $G$-espaço $X$ é G-invariante se $g A=A$ para todo $g \in G$.

Denote por $X / G$ o conjunto cujos elementos são as órbitas $G(x)$ em $X$. Considere a aplicação natural $X \rightarrow X / G$ que leva cada elemento $x \in X$ em sua órbita $G(x)$. Então $X / G$ munido da topologia quociente é chamado espaço de órbitas de $X$ (com respeito a $G)$.

OBSERVAÇÃo 1.1.1. Em geral $X / G$ não é uma variedade topológica. (Vide [tD], Exercício 3, p.19).

O próximo resultado pode ser encontrado em ([Br], Proposição 4.1, p.40).

Proposição 1.1.2. Se $G$ é compacto, então a aplicação $G / G_{x} \rightarrow G(x)$ definida por $g G_{x} \mapsto g x$ é um homeomorfismo.

Para todo subgrupo $H$ de $G$, denotaremos por $N H$ o normalizador de $H$ em $G$ e por $W H=N H / H$ o grupo de Weyl de $H$ em $G$.

A classe de conjugação de $H$ será denotada por $(H)$ e será chamada órbita do tipo $H$. Um subgrupo $K$ de $G$ é um subconjugado de $H$, se existir $g \in G$ tal que $g K g^{-1}$ é um subgrupo de $H$. Uma órbita do tipo $H$ é chamada um tipo de isotropia de $X$ se $H$ aparece como um subgrupo de isotropia de algum ponto $x \in X$. Note que se $K \in(H)$, então $K=g H^{-1}$, para algum $g \in G$ e assim, $K$ aparece como o subgrupo de isotropia de $g x \in X$. Isto ocorre pois $G_{g x}=g G_{x} g^{-1}$, para todo $x \in X$ e $g \in G$ (vide $[\mathrm{Br}]$, p.35).

Para cada subgrupo $H$ de $G$, 
(1) $X_{H}=\left\{x \in X \mid G_{x}=H\right\}$ é o conjunto dos pontos de isotropia $H$.

(2) $X_{(H)}=G X_{H}=\left\{x \in X \mid\left(G_{x}\right)=(H)\right\}$.

(3) $X^{H}=\{x \in X \mid h x=x, \forall h \in H\}=\left\{x \in X \mid H \subset G_{x}\right\}$ é chamado o conjunto dos pontos fixos por $\mathrm{H}$.

(4) $X^{(H)}=G X^{H}=\left\{x \in X^{K} \mid K \in(H)\right\}$.

A demonstração do teorema a seguir pode ser encontrada em ([Ka], Teorema 1.46, p.31).

Teorema 1.1.3. Se $X$ é um G-espaço Hausdorff, então $X^{H}$ é fechado em $X$ para qualquer $H \subset G$.

Se $H_{i}$ é subconjugado a $H_{j}$ escrevemos $\left(H_{i}\right) \leq\left(H_{j}\right)$. Suponha que $X$ tenha um conjunto finito de tipos de isotropias $\left\{\left(H_{i}\right)\right\}_{i=1}^{k}$. Podemos definir uma ordenação admissível em $\left\{\left(H_{i}\right)\right\}_{i=1}^{k}$ tal que $\left(H_{j}\right) \leq\left(H_{i}\right)$ implica $i \leq j$ (vide [Ka], Lema 1.80, p.51). Existe, então, uma filtração associada $X_{1} \subset X_{2} \subset \ldots \subset X_{k}=X$ de $G$-espaços $X_{i}$, onde $X_{1}=X_{\left(H_{1}\right)}, X_{i}=\left\{x \in X \mid\left(G_{x}\right)=\left(H_{j}\right)\right.$ para algum $\left.j \leq i\right\}$, além disso, temos a seguinte igualdade $X_{\left(H_{i}\right)}=X_{i}-X_{i-1}$.

\section{$1.2 \quad G$-Espaço Induzido}

Sejam $G$ um grupo topológico e $H$ um subgrupo de $G$. Dado um $H$-espaço $X$, podemos construir um $G$-espaço associado a $X$ como segue.

Construção. Como $X$ é um $H$-espaço, defina uma $H$-ação em $G \times X$ por $(h,(g, x)) \mapsto$ $\left(g h^{-1}, h x\right)$. O espaço de órbitas desta $H$-ação é denotado por $G \times_{H} X$. A $H$-órbita de $(g, x)$ será denotada por $[g, x]$. Defina a $G$-ação

$$
\begin{array}{ccc}
G \times G \times_{H} X & \longrightarrow G \times_{H} X \\
\left(g^{\prime},[g, x]\right) & \longmapsto & {\left[g^{\prime} g, x\right]}
\end{array}
$$


A $G$-ação em $G \times_{H} X$ definida acima é chamada G-ação induzida e $G \times_{H} X$ é chamado de G-espaço induzido.

O próximo resultado pode ser encontrado em ([Ka], Lema 1.93, p.60).

Lema 1.2.1. Sejam $G$ um grupo compacto e $X$ um $\mathrm{T}_{1}$, G-espaço. Se $X$ possui um único tipo de isotropia $(H)$, então $G_{x}=H$ para todo $x \in X^{H}$. Ou seja, $X_{H}=X^{H}$.

Corolário 1.2.2. Nas condições do lema anterior, $W H$ atua livremente em $X^{H}$.

O teorema a seguir pode ser encontrado em ([Br], p.89). Daremos uma idéia da demonstração do corolário (1.2.6), pois a extensão de uma $W H$-aplicação para uma G-aplicação será usada na proposição (3.2.4) do capítulo 3.

Teorema 1.2.3. Sejam $G$ um grupo compacto e $X$ um G-espaço Hausdorff. Se $X$ tem um único tipo de isotropia $(H)$, então a aplicação $G \times_{N H} X^{H} \rightarrow X$, dada por $[g, x] \mapsto g x$, é um G-homeomorfismo.

Corolário 1.2.4. Nas condições do teorema anterior, a inclusão $X^{H} \subset X$ induz um homeomorfismo $X^{H} / N \rightarrow X / G$.

Corolário 1.2.5. Nas condições do teorema (1.2.3), a aplicação $(G / H) \times_{W H} X^{H} \rightarrow X$, definida por $[g H, x] \mapsto g x$, é um G-homeomorfismo.

Corolário 1.2.6. Com as hipóteses de (1.2.3), seja $Y$ um G-espaço. Existe uma correspondência entre $G$-aplicações $X \rightarrow Y$ eWH-aplicações $X^{H} \rightarrow Y^{H}$.

Demonstração. Seja $f: X^{H} \rightarrow Y^{H}$ uma $W H$-aplicação. Defina $\psi:(G / H) \times_{W H}$ $X^{H} \rightarrow(G / H) \times_{W H} Y^{H}$ por $[g H, x] \mapsto[g H, f(x)]$. Pelo corolário (1.2.5), as aplicações $\xi: X \rightarrow(G / H) \times_{W H} X^{H}$ e $\rho:(G / H) \times_{W H} Y^{H} \rightarrow Y$ são $G$-homeomorfismos. Agora, defina $f^{\prime}: X \rightarrow Y$ como a composição $\rho \psi \xi$. Então, para $x \in X^{H}, f^{\prime}(g x)=g f(x)$ tal que $f^{\prime} \mid X^{H}=f$. 


\section{$1.3 \quad G$-Fibração}

Agora, vejamos a definição de $G$-homotopia. Dizemos que as $G$-aplicações $f_{0}, f_{1}: X \rightarrow$ $Y$ são G-homotópicas se existe uma $G$-aplicação, chamada G-homotopia de $f_{0}$ a $f_{1}, F: X \times I \rightarrow Y$ tal que $F(x, 0)=f_{0}(x), F(x, 1)=f_{1}(x)$ e $g F(x, t)=F(g x, t)$, para todo $x \in X$ e $t \in I$. A $G$-ação em $I=[0,1]$ é trivial.

Seja $p: E \rightarrow B$ uma $G$-aplicação. Dizemos que $p$ possui a G-Propriedade do Levantamento de Homotopia $(G$-PLH) se, dado um diagrama comutativo

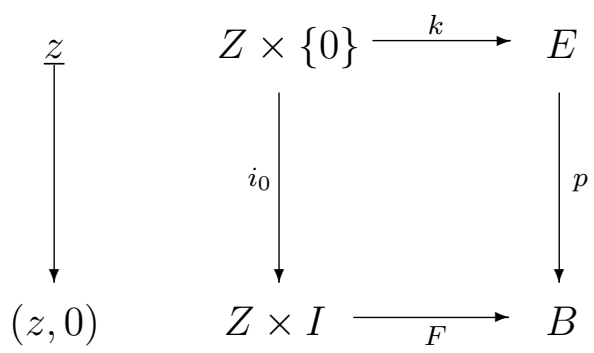

de $G$-aplicações, existe uma $G$-aplicação $\bar{F}: Z \times I \rightarrow E$ tal que $p \bar{F}=F$ e $\bar{F} i_{0}=k$. Uma G-fibração é uma $G$-aplicação $p: E \rightarrow B$ satisfazendo a $G$-PLH para todo G-espaço $Z$.

\subsection{Teoria de Nielsen de Raízes}

Seja $f: X \rightarrow Y$ uma aplicação entre espaços topológicos e seja $a \in Y$ um ponto arbitrário. Uma raiz da equação $f(x)=a$ é um ponto $x_{0} \in X$ tal que $f\left(x_{0}\right)=a$.

Definição 1.4.1. Duas raízes $x_{0}$ e $x_{1}$ de $f$ em a são Nielsen equivalentes, $x_{0} \sim x_{1}$, se existe um caminho $c: I \rightarrow X$ de $x_{0}$ para $x_{1}$ tal que $f c \simeq \bar{a} \operatorname{rel}\{0,1\}$, onde $\bar{a}$ denota o caminho constante em $a$.

Esta relação é de equivalência e separa o conjunto das raízes em subconjuntos disjuntos, chamados classes de Nielsen de raízes de $f$ em $a$. O próximo resultado é demonstrado em ([K], Teorema 3.4, p.126) e em ([B3], Corolário 3.6, p.380). 
Lema 1.4.2. Seja $f: X \rightarrow Y$ e a $\in$ Y. Se $X$ é compacto então existe apenas um número finito de classes de raizes. Além disso, toda classe de raízes é compacta.

Agora, seja $\left\{f_{t}: X \rightarrow Y\right\}$ uma homotopia começando em $f$. A próxima definição relaciona as raízes de $f=f_{0}$ com as raízes de $f_{1}$. Dizemos que uma raiz $x_{0}$ de $f$ em $a$ está $\left\{f_{t}\right\}$-relacionada com a raiz $x_{1}$ de $f_{1}$ em $a$, denotada por $x_{0}\left\{f_{t}\right\} x_{1}$, se existe um caminho $c$ em $X$ de $x_{0}$ para $x_{1}$ tal que o laço $\left\{f_{t} \circ c(t)\right\}$ é homotópico, com extremos fixos, ao caminho constante em $a$. Logo, se $x_{0}, x_{1} \in f^{-1}(a)$ então $x_{0} \sim x_{1}$ se, e somente se, $x_{0}$ está relacionado a $x_{1}$ pela homotopia constante em $f$. Pode-se mostrar (vide [K], Teorema 4.2, p.128) que se $\left\{f_{t}\right\}$ é uma homotopia começando em $f$ e se uma raiz em um classe de raízes $\alpha$ de $f$ está $\left\{f_{t}\right\}$-relacionada a uma raiz da classe $\alpha_{1}$ de $f_{1}$, então qualquer raiz em $\alpha$ está $\left\{f_{t}\right\}$-relacionada à uma raiz de $\alpha_{1}$. Em outras palavras, a relação $x_{0}\left\{f_{t}\right\} x_{1}$ induz uma correspondência de $\alpha$ para $\alpha_{1}$ sob $\left\{f_{t}\right\}$, que é denotada por $\alpha\left\{f_{t}\right\} \alpha_{1}$.

Definição 1.4.3. Se uma classe de raízes $\alpha$ de $f$ corresponde a uma classe de raízes $\alpha_{1}$ de $f_{1}$, ou seja $\alpha\left\{f_{t}\right\} \alpha_{1}$, para toda homotopia $\left\{f_{t}\right\}$ começando em $f$, então a é chamada essencial.

Em outras palavras, uma classe de raízes é inessencial se ela "desaparece" por alguma homotopia de $f$. O número de classes de raízes essenciais é chamado número de Nielsen de raízes de $f$ em a e será denotado por $N(f, a)$.

Se $Y$ é uma variedade, então o número de Nielsen de raízes $N(f, a)$ independe do ponto $a \in Y$, isto é, $N(f, a)=N(f, b)$ para todo $a, b \in Y$. (Ver [B3], Corolário 2.22, p.388).

O próximo resultado pode ser encontrado em ([K], Teorema 4.4, p.129).

Lema 1.4.4. O número de Nielsen de raizes é um invariante homotópico, isto é, se $f$ e g são aplicações homotópicas, então $N(f, a)=N(g, a)$.

Note que $N(f, a) \leq M R[f, a]$, onde $M R[f, a]=\min \left\{\# \mathrm{~g}^{-1}(\mathrm{a}) \mid \mathrm{g} \in[f]\right\}$. Aqui, [ ] significa classe de homotopia e $\# g^{-1}(a)$ denota o número de elementos de $g^{-1}(a)$. Observe ainda que, pelo lema (1.4.2), segue que $0 \leq N(f, a)<\infty$. 
Agora, veremos uma interpretação de Nielsen equivalência e $\left\{f_{t}\right\}$-relação usando espaços de revestimento.

A aplicação $f$ induz um homomorfismo $f_{\#}: \pi_{1}(X) \rightarrow \pi_{1}(Y)$ de grupos fundamentais. Como a imagem de $f_{\#}$ é um subgrupo de $\pi_{1}(Y)$, existe uma aplicação de revestimento $\eta: \widehat{Y} \rightarrow Y$ tal que $\eta_{\#} \pi_{1}(\widehat{Y})=f_{\#} \pi_{1}(X)$. Logo, podemos levantar $f$ através de $\eta$ para $\hat{f}: X \rightarrow \widehat{Y}$ fazendo o seguinte diagrama comutar.

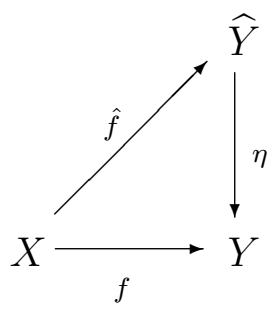

A aplicação $\hat{f}$ será chamada levantamento de Hopf de $f$ e $\eta$ é um revestimento de Hopf para $f$. Note que se $\eta: \widehat{Y} \rightarrow Y$ é um revestimento de Hopf para $f$, então $\eta$ também é um revestimento de Hopf para qualquer aplicação homotópica a $f$.

O próximo teorema pode ser encontrado em ([B2], Lema 1 e Lema 2) e em ([B3], Teorema 3.4, p.379).

Teorema 1.4.5. Sejam $f: X \rightarrow Y$ uma aplicação, a $\in Y$ um ponto arbitrário, $\eta: \widehat{Y} \rightarrow Y$ um revestimento de Hopf para $f$ e $\hat{f}: X \rightarrow \widehat{Y}$ levantamento de Hopf de $f$ por $\eta$. Suponha $\left\{f_{t}: X \rightarrow Y\right\}$ uma homotopia começando em $f$ e seja $\left\{\hat{f}_{t}: X \rightarrow \widehat{Y}\right\}$ seu levantamento através de $\eta$ começando em $\hat{f}$. Então

(1) As raizes $x_{0}, x_{1} \in f^{-1}(a)$ são Nielsen equivalentes se, e somente se, $\hat{f}\left(x_{0}\right)=$ $\hat{f}\left(x_{1}\right)$.

(2) Uma raiz $x_{0} \in f^{-1}(a)$ está $\left\{f_{t}\right\}$-relacionada a uma raiz $x_{1}$ de $f_{1}$ em a se, e somente se, $\hat{f}\left(x_{0}\right)=\hat{f}_{1}\left(x_{1}\right)$.

O teorema acima diz que, para cada $\hat{y} \in \hat{\eta}^{-1}(a)$, o conjunto $\hat{f}^{-1}(\hat{y})$ é uma classe de Nielsen de raízes, se for não-vazio. Além disso, a classe de raízes $\hat{f}^{-1}(\hat{y})$ é essencial se, e somente se, $\hat{f}_{1}^{-1}(\hat{y}) \neq \emptyset$ para toda aplicação $\hat{f}_{1}: X \rightarrow \widehat{Y}$ homotópica a $\hat{f}$. 
A seguir, veremos a definição do número de Reidemeister de raízes e que este número constitui um limitante superior para o número de Nielsen de raízes.

No teorema (1.4.5), vimos que o conjunto das classes de raízes está em correspondência com um subconjunto de $\eta^{-1}(a)$ e, portanto, está em correspondência com um subconjunto de $\eta^{-1}(y)$ para qualquer $y \in Y$. Pode-se mostrar, usando a teoria de espaços de revestimento, que para qualquer $x \in X$, o grupo $\pi_{1}(Y, f(x))$ atua transitivamente na fibra de $f(x)$ e o grupo de isotropia do ponto $\hat{f}(x) \in \widehat{Y}$ é $\eta_{\#} \pi_{1}(\widehat{Y}, \hat{f}(x))$. Então a cardinalidade de $\eta^{-1}(f(x))$ é o índice do subgrupo $\eta_{\#} \pi_{1}(\widehat{Y}, \hat{f}(x))$ em $\pi_{1}(Y, f(x))$.

Como $\eta$ é um revestimento de Hopf para $f$, então $\eta_{\#} \pi_{1}(\widehat{Y}, \hat{f}(x))=f_{\#} \pi_{1}(X, x)$, e portanto, o conjunto das classes de Nielsen de raízes está em correspondência com um subconjunto do conjunto de classes laterais $\pi_{1}(Y, f(x)) / f_{\#} \pi_{1}(X, x)$. O número destas classes laterais é, portanto, um limitante superior para o número de classes de raízes, e conseqüentemente, é um limitante superior para $N(f, a)$, o número de classes de raízes essenciais.

A discussão acima motiva a seguinte definição (vide [B1]).

Definição 1.4.6. Seja $f: X \rightarrow Y$ uma aplicação e seja a $\in Y$. O número de Reidemeister de raízes de $f$ em a é definido por $R(f, a):=\left[\pi_{1}(Y): f_{\#} \pi_{1}(X)\right]=$ $\# \eta^{-1}(a)$.

O próximo teorema é uma ferramenta importante para o cálculo do número de Nielsen de raízes. (Ver [B1] ou ([B3], p.388)).

Teorema 1.4.7. Suponha $f: X \rightarrow Y$ uma aplicação, $X$ um espaço compacto, $Y$ uma variedade e $a \in Y$. Então $N(f, a)=0$ ou $N(f, a)=R(f, a)$. Conseqüentemente, se $R(f, a)=\infty$ ent $\tilde{a} o N(f, a)=0$.

Agora, considere uma classe de raízes $\alpha$ de $f$ em $a$. O índice de raiz de $\alpha$ com respeito a $f$ é o homomorfismo em homologia singular $\omega(f, \alpha)=f_{*} \circ e_{*}^{-1} \circ i_{*}: H_{*}(X) \rightarrow$ $H_{*}(Y, Y-a)$ induzido por

$$
X \stackrel{i}{\hookrightarrow}(X, X-\alpha) \stackrel{e}{\longleftarrow}(N, N-\alpha) \stackrel{f}{\longrightarrow}(Y, Y-a)
$$


onde $N$ é uma vizinhança fechada de $\alpha$ que não contém outras raízes de $f$, exceto as de $\alpha$. A aplicação e é uma excisão e $H_{*}(X)$ denota $\bigoplus_{k=0} H_{k}(X)$. Deste modo, R. Brooks provou o seguinte resultado (vide [B1], Corolário 2, Teorema 2).

Teorema 1.4.8. Sejam $X$ e $Y$ variedades compactas conexas (não necessariamente de mesma dimensão) com $Y$ orientável e seja $a \in Y$. Para quaisquer classes de raízes $\alpha$ e $\beta$ de $f$ em a, temos então que $\omega(f, \alpha)=\omega(f, \beta)$. Além disso, se $f$ possui uma classe de raízes essencial, então todas as classes de raízes são essenciais e $N(f, a)=R(f, a)$.

OBSERVAÇÃo 1.4.9. A recíproca deste teorema não é verdadeira. A aplicação de Hopf $p: S^{3} \rightarrow S^{2}$ possui uma única classe de raízes, a qual é essencial com índice zero. De fato, como $S^{2}$ é simplesmente conexa, então todas as raízes de $p$ estão na mesma classe de raízes $\alpha$, cujo índice $\omega(p, \alpha)$ é o homomorfismo nulo. A demonstração de que $p$ não é homotópica a uma aplicação constante pode ser encontrada em ([Hu], Proposição 5.1, p.67). 


\section{Capítulo 2}

\section{Teoria de Nielsen Equivariante}

Neste capítulo, descrevemos parte da teoria de Nielsen de raízes para aplicações equivariantes desenvolvida por P. Wong, em seu importante artigo de 1999 [W4]. Em todo este capítulo iremos considerar $X$ e $Y G$-espaços, com as propriedades descritas no capítulo 1. Sejam $f: X \rightarrow Y$ uma $G$-aplicação, $a \in Y^{G}=\{y \in Y \mid g y=y, \forall g \in G\} \neq \emptyset \mathrm{e}$ $f^{-1}(a) \neq \emptyset$.

Após uma breve exposição sobre $G$-classes de raízes na seção 2.1, o conceito de $G$-número de Nielsen de raízes, $N_{G}(f, a)$, é definido na seção 2.2, onde também serão apresentados alguns exemplos dos números de Nielsen $N(f, a)$ e $N_{G}(f, a)$. Na seção 2.3, definimos uma $G$-ação no espaço de revestimento de Hopf $\widehat{Y}$ de $Y$, o que tornará o revestimento de Hopf $\eta: \widehat{Y} \rightarrow Y$ para $f$ e o levantamento $\hat{f}: X \rightarrow \widehat{Y}$ (e, portanto, todos os levantamentos) de $f$ aplicações equivariantes.

Uma versão equivariante do teorema (1.4.5) será demonstrada na seção 2.3. A seção 2.5 apresenta uma definição do $G$-número de Reidemeister de raízes, abordando o conceito de revestimento de Hopf. Tal definição difere da original, dada por P. Wong em [W4], onde foi usado espaços de revestimento universais de $X$ e de $Y$. 


\section{$2.1 \quad G$-Classes de Nielsen de raízes}

No que faremos a seguir, vamos utilizar o conceito de $G$-classe de Nielsen de raízes apresentado em [W4]. Primeiramente, observemos que se $x_{0}$ é uma raiz da $G$-aplicação $f$ em $a \in Y^{G} \neq \emptyset$, então os elementos da órbita de $x_{0}, G\left(x_{0}\right)$, também são raízes de $f$. De fato, $f\left(g x_{0}\right)=g f\left(x_{0}\right)=g a=a$, para qualquer $g \in G$.

Definição 2.1.1. Dizemos que duas raízes $x_{0}$ e $x_{1}$ de $f$ em a são G-Nielsen equivalentes se

(i) $x_{0}=g x_{1}$, para algum $g \in G$ ou

(ii) Existe um caminho $c: I \rightarrow X$ de $x_{0}$ para $g x_{1}$, para algum $g \in G$, tal que $f c \simeq \bar{a}$ rel $\{0,1\}$, onde $\bar{a}$ é o caminho constante em a.

Lema 2.1.2. A G-Nielsen relação definida acima é uma relação de equivalência e será denotada por $x_{0} \sim_{G} x_{1}$. Logo $f^{-1}(a)$ é separado em subconjuntos disjuntos.

DemonstraÇÃO. Sejam $x, y, z \in f^{-1}(a)$.

(1) (Reflexiva) $x \sim_{G} x, \forall x \in f^{-1}(a)$, pois $x=e x$.

(2) (Simétrica) Suponha que $x \sim_{G} y$. Então temos dois casos a considerar.

(i) $x=g y$ para algum $g \in G$. Então $y=g^{-1} x$.

(ii) Existe um caminho $c: I \rightarrow X$ de $x$ a $g y$, para algum $g \in G$, tal que $f c \simeq \bar{a}$ rel $\{0,1\}$. Considere o caminho $d=g^{-1} c^{-1}: I \rightarrow X$, que é dado por $d(t)=$ $g^{-1} c(1-t)$. Logo, $d(0)=g^{-1} c(1)=\left(g^{-1} g\right) y=y, d(1)=g^{-1} c(0)=g^{-1} x$ e $f(d(t))=f\left(g^{-1} c(1-t)\right)=g^{-1} f(c(1-t)) \simeq g^{-1} \bar{a}(1-t)=g^{-1} a=a$.

Portanto, em ambos os casos, temos que $y \sim_{G} x$.

(3) (Transitiva) Suponha que $x \sim_{G} y$ e $y \sim_{G} z$. Temos quatro possibilidades. 
(i) Se $x=g_{1} y$ e se $y=g_{2} z$, para alguns $g_{1}, g_{2} \in G$, então $x=\left(g_{1} g_{2}\right) z$. Logo, $x \sim_{G} z$.

(ii) Se $x=g_{1} y$, para algum $g_{1} \in G$, e se existe um caminho $c: I \rightarrow X$ de $y$ para $g_{2} z$, para algum $g_{2} \in G$, tal que $f c \simeq \bar{a} \operatorname{rel}\{0,1\}$, então considere o caminho $d(t)=g_{1} c(t)$ em $X$. Note que $d$ começa em $x$, termina em $g_{1} g_{2} z$ e $f d \simeq \bar{a}$ $\operatorname{rel}\{0,1\}$. Assim, $x \sim_{G} z$.

(iii) Se existe um caminho $c: I \rightarrow X$ de $x$ para $g_{1} y$, para algum $g_{1} \in G$, tal que $f c \simeq \bar{a} \operatorname{rel}\{0,1\}$ e se $y=g_{2} z$, para algum $g_{2} \in G$, então o próprio caminho $c$ de $x$ a $\left(g_{1} g_{2}\right) z$ satisfaz a definição de $G$-Nielsen equivalência. Logo, $x \sim_{G} z$.

(iv) Se existirem caminhos $c, d: I \rightarrow X \operatorname{com} c(0)=x, c(1)=g_{1} y, d(0)=y$ e $d(1)=g_{2} z$, para alguns $g_{1}, g_{2} \in G$, tais que $f c \simeq \bar{a} \operatorname{rel}\{0,1\}$ e $f d \simeq \bar{a}$ $\operatorname{rel}\{0,1\}$, então o caminho $h=c * g_{1} d: I \rightarrow X$ dado por

$$
h(t)=\left(c * g_{1} d\right)(t)=\left\{\begin{array}{l}
c(2 t), 0 \leq t \leq 1 / 2 \\
g_{1} d(2 t-1), 1 / 2 \leq t \leq 1
\end{array}\right.
$$

satisfaz $h(0)=x, h(1)=g_{1} g_{2} z$ e $f h \simeq \bar{a} \operatorname{rel}\{0,1\}$. Portanto, $x \sim_{G} z$.

As classes de equivalência dadas pelo lema acima, em $f^{-1}(a)$, são chamadas Gclasses de Nielsen de raízes de $f$ em $a$.

Segue da definição (2.1.1) que, se $\alpha$ é uma classe de raízes de $f$ (esquecendo a $G$ equivariância), então existe uma única $G$-classe de raízes $\mathcal{R}$ tal que $\alpha \subset \mathcal{R}$. Se $X$ é compacto, então pelo lema (1.4.2), o número de classes de raízes é finito. Logo, existe somente um número finito de $G$-classes de raízes. Portanto, $f^{-1}(a)$ é particionado em $G$-classes de raízes, disjuntas duas a duas, $\mathcal{R}_{1}, \ldots, \mathcal{R}_{m}$, onde cada $G$-classes de raízes é uma união disjunta de classes de raízes. Por outro lado, $\mathcal{R}_{j}$ é uma união disjunta de $G$-órbitas de raízes, para cada $j=1, \ldots, m$. 


\section{$2.2 \quad G$-Número de Nielsen de Raízes}

O objetivo desta seção é definir, conforme [W4], o G-número de Nielsen de raízes e mostrar sua invariância por $G$-homotopias.

Seja $\left\{f_{t}: X \rightarrow Y\right\}$ uma $G$-homotopia começando em $f$.

Definição 2.2.1. Uma raiz $x_{0}$ de $f$ em a está $\left\{f_{t}\right\}_{G}$-relacionada a uma raiz $x_{1}$ de $f_{1}$ em $a, x_{0}\left\{f_{t}\right\}_{G} x_{1}$, se existe um caminho $c: I \rightarrow X$ de $x_{0}$ para gx $x_{1}$, para algum $g \in G$ tal que o laço $\left\{f_{t} \circ c(t)\right\}$ é homotópico, com extremos fixos, ao caminho constante em $a$, isto é, $\bar{a}$.

Portanto, se $x_{0}\left\{f_{t}\right\}_{G} x_{1}$ então $x_{0}\left\{f_{t}\right\} g x_{1}$, para algum $g \in G$.

Assim como no caso não-equivariante, a $\left\{f_{t}\right\}_{G}$-relação, dada pela definição acima, induz uma correspondência entre as $G$-classes de raízes de $f=f_{0}$ e de $f_{1}$. Este fato será provado a seguir.

Lema 2.2.2. Sejam $x_{0} \in f^{-1}(a)$ e $x_{1} \in f_{1}^{-1}(a)$ tais que $x_{0}\left\{f_{t}\right\}_{G} x_{1}$. Considere as $G$-classes de raízes $\mathcal{R}_{0}$ de $f$ e $\mathcal{R}_{1}$ de $f_{1}$ contendo $x_{0}$ e $x_{1}$, respectivamente. Então

(1) $x_{0}^{\prime} \in \mathcal{R}_{0} \Leftrightarrow x_{0}^{\prime}\left\{f_{t}\right\}_{G} x_{1}$.

(2) $x_{1}^{\prime} \in \mathcal{R}_{1} \Leftrightarrow x_{0}\left\{f_{t}\right\}_{G} x_{1}^{\prime}$.

Demonstração. Segue da definição (2.1.1) que existe uma única classe de raízes $\alpha_{i}$ em $\mathcal{R}_{i}$ tal que $x_{i} \in \alpha_{i}, i=0,1$. Como $x_{0}\left\{f_{t}\right\}_{G} x_{1}$ então existe um caminho $c: I \rightarrow X$ de $x_{0}$ para $h x_{1}$, para algum $h \in G$, tal que $\left\{f_{t} \circ c(t)\right\} \simeq \bar{a} \operatorname{rel}\{0,1\}$, ou seja, $x_{0}\left\{f_{t}\right\} h x_{1}$.

(1) $(\Rightarrow)$ Como $x_{0}$ e $x_{0}^{\prime} \in \mathcal{R}_{0}$, temos duas possibilidades.

(i) $x_{0}=g x_{0}^{\prime}$, para algum $g \in G$. Neste caso, considere o caminho $g^{-1} c: I \rightarrow X$ de $g^{-1} x_{0}=x_{0}^{\prime}$ para $\left(g^{-1} h\right) x_{1}$. Logo, $\left\{f_{t} \circ g^{-1} c(t)\right\} \simeq \bar{a} \operatorname{rel}\{0,1\}$. Então $x_{0}^{\prime}\left\{f_{t}\right\}_{G} x_{1}$. 
(ii) Se existe um caminho $d: I \rightarrow X$ de $x_{0}$ para $g x_{0}^{\prime}$, para algum $g \in G$, tal que $f \circ d \simeq \bar{a} \operatorname{rel}\{0,1\}$, então $g x_{0}^{\prime} \in \alpha_{0}$. Como $x_{0}\left\{f_{t}\right\} h x_{1}$, então $g x_{0}^{\prime}\left\{f_{t}\right\} h x_{1}$. Portanto $x_{0}^{\prime}\left\{f_{t}\right\}_{G} x_{1}$.

(1) $(\Leftarrow)$ Como $x_{0}^{\prime}\left\{f_{t}\right\}_{G} x_{1}$, então $x_{1}\left\{f_{t}^{-1}\right\} g x_{0}^{\prime}$, para algum $g \in G$. Por outro lado, $x_{1}\left\{f_{t}^{-1}\right\} h^{-1} x_{0}$, logo $g x_{0}^{\prime}$ e $h^{-1} x_{0}$ pertencem a mesma classe de raízes de $f$, ou seja , $g x_{0}^{\prime} \sim h^{-1} x_{0}$. Mas $g x_{0}^{\prime}, x_{0}^{\prime} \in G\left(x_{0}^{\prime}\right)$ e $x_{0}, h^{-1} x_{0} \in G\left(x_{0}\right) \subset \mathcal{R}_{0}$. Portanto $x_{0}^{\prime} \in \mathcal{R}_{0}$.

Analogamente, pode-se provar (2).

Vamos denotar por $\mathcal{R}_{0}\left\{f_{t}\right\}_{G} \mathcal{R}_{1}$ a correspondência entre as $G$-classes de raízes $\mathcal{R}_{0}$ e $\mathcal{R}_{1}$.

Definição 2.2.3. Uma $G$-classe de raízes $\mathcal{R}$ de $f$ é essencial se dada qualquer $G$ homotopia $\left\{f_{t}: X \rightarrow Y\right\}$ começando em $f, \mathcal{R}$ está $\left\{f_{t}\right\}_{G}$-relacionada a uma $G$-classe de raízes de $f_{1}$. Caso contrário, $\mathcal{R}$ é chamada inessencial.

O G-número de Nielsen de raizes de $f$ em a é definido como sendo o número de $G$-classes de raízes essenciais e será denotado por $N_{G}(f, a)$.

Se $X$ é um espaço compacto, segue da definição (2.1.1) e do lema (1.4.2) que $0 \leq N_{G}(f, a)<\infty$.

Agora, observe que, \#\{G-classes de raízes $\} \leq \#\{$ classes de raízes $\}$ Entretanto, esta desigualdade não é verdadeira se considerarmos $G$-classes de raízes essenciais e classes de raízes essenciais, conforme veremos no exemplo (2.2.4).

Exemplo 2.2.4. Vide [W3], Exemplo 7.1, p.41.

Considere $S^{2}=S^{3} / S^{1}$ como um espaço de classes e seja $[e] \in S^{2}$ a classe do elemento identidade e no grupo de Lie compacto conexo $S^{3}$. Suponha $h=i d: S^{2} \rightarrow S^{2}$ a aplicação identidade e $f: S^{3} \rightarrow S^{2}$ a aplicação dada por $f(z)=z^{-1} h\left(z S^{1}\right)=e S^{1}=[e]$, ou seja, $f$ é a aplicação constante em $[e]$. 
Considere a $S^{1}$-ação livre $k \cdot z=z k^{-1}$ em $S^{3}$ e a $S^{1}$-ação (não-livre) $k * z S^{1}=(k z) S^{1}$ em $S^{2}$.

Como $f$ é uma aplicação constante e $S^{2}$ é um espaço simplesmente conexo, segue que $S^{3}$ é a única classe de raízes de $f$, a qual é inessencial. Logo $N(f,[e])=0$.

Agora, observe que, $f$ é uma $S^{1}$-aplicação. Pelo Lema (1.2) de [W5], $\omega_{S^{1}}\left(f, S^{3}\right)=$ $L(h)$, onde $\omega_{S^{1}}\left(f, S^{3}\right)$ é um número inteiro chamado índice de raiz equivariante da $S^{1}$-classe de raízes $S^{3}$, com respeito a $f$, o qual é definido em [W3]. De modo usual, $L(h)$ denota número de Lefschetz de $h$. Como $\omega_{S^{1}}\left(f, S^{3}\right) \neq 0$, pois $L(h)=2$, então a $S^{1}$-classe de raízes $S^{3}$ é essencial. Portanto $N_{S^{1}}(f,[e])=1$.

Exemplo 2.2.5. Sejam $\mathbb{R} P^{2}$ o plano projetivo e $f: S^{2} \rightarrow \mathbb{R} P^{2}$ a aplicação identificação. Denote por $n=(0,0,1)$ e $s=(0,0,-1)$ os pólos norte e sul, respectivamente, de $S^{2}$ e seja $a=\{n, s\}=f(\{n, s\}) \in \mathbb{R} P^{2}$. Então as únicas raízes de $f$ em $a$ são $n$ e $s$.

Seja $c$ um caminho em $S^{2}$ de $n$ a $s$. Logo, $f c$ é um laço em $a$, cuja classe de homotopia com extremos fixos é um gerador do grupo fundamental $\pi_{1}\left(\mathbb{R} P^{2}, a\right) \simeq \mathbb{Z} / 2 \mathbb{Z}$. Assim, $n$ e $s$ não são Nielsen equivalentes, ou seja, $f$ possui exatamente duas classes de raízes, a saber, $\{n\}$ e $\{s\}$. Agora, seja $N$ uma vizinhança fechada de $n$ contida no hemisfério norte aberto de $S^{2}$ e seja $f^{\prime}:(N, N-n) \rightarrow(f(N), f(N)-a)$ uma aplicação induzida por $f$. Considere as aplicações

$$
S^{2} \stackrel{i}{\hookrightarrow}\left(S^{2}, S^{2}-n\right) \stackrel{e}{\hookrightarrow}(N, N-n) \stackrel{f^{\prime}}{\rightarrow}(f(N), f(N)-a) \stackrel{e^{\prime}}{\hookrightarrow}\left(\mathbb{R} P^{2}, \mathbb{R} P^{2}-a\right)
$$

onde $e, e^{\prime}$ são excisões. Como $f$ é uma aplicação de revestimento, $f^{\prime}$ é um homeomorfismo. Logo, $e, e^{\prime}$ e $f^{\prime}$ induzem isomorfismos em homologia (estamos considerando os coeficientes em $\mathbb{Z}$ ). Agora, note que, a inclusão $i$ induz um isomorfismo na dimensão 2. Portanto o índice de raiz

$$
\omega_{2}(f,\{n\} ; \mathbb{Z})=e_{2}^{\prime} \circ f_{2}^{\prime} \circ e_{2}^{-1} \circ i_{2}: H_{2}\left(S^{2} ; \mathbb{Z}\right) \rightarrow H_{2}\left(\mathbb{R} P^{2}, \mathbb{R} P^{2}-a ; \mathbb{Z}\right)
$$

é um isomorfismo não-trivial. Assim, concluímos que a classe de raiz $\{n\}$ é essencial. Analogamente prova-se também que $\{s\}$ é essencial, e portanto, $N(f, a)=2$. 
Agora, considere a ação antípoda de $\mathbb{Z}_{2}$ em $S^{2}$ e a $\mathbb{Z}_{2}$-ação trivial em $\mathbb{R} P^{2}$. Então $f$ é equivariante e o conjunto de pontos-fixos por $\mathbb{Z}_{2}$ é $\left(\mathbb{R} P^{2}\right)^{\mathbb{Z}_{2}}=\mathbb{R} P^{2}$. Como as raízes $n$ e $s$ de $f$ em $a$ estão na mesma órbita, $f$ possui uma única $\mathbb{Z}_{2}$-classe de raízes $\mathcal{R}=\{n\} \cup\{s\}$. Portanto, $\mathcal{R}$ é essencial, pois as classes de raízes $\{n\}$ e $\{s\}$ são essenciais; $\log \mathrm{o} N_{\mathbb{Z}_{2}}(f, a)=1$.

O próximo lema mostra que se $f$ e $f^{\prime}$ são $G$-aplicações $G$-homotópicas, então $N_{G}(f, a)=N_{G}\left(f^{\prime}, a\right)$.

Lema 2.2.6. $O$ G-número de Nielsen de raízes, $N_{G}(f, a)$, é um invariante por $G$ homotopias.

DemonstraçÃo. Seja $\left\{f_{t}: X \rightarrow Y\right\}$ uma $G$-homotopia começando em $f$ e seja $\mathcal{R}$ uma $G$-classe de raízes essencial de $f$. Então existe uma $G$-classe de raízes $\mathcal{R}_{1}$ de $f_{1}$ em $a$ tal que $\mathcal{R}\left\{f_{t}\right\}_{G} \mathcal{R}_{1}$. Pelo lema (2.2.2), $\mathcal{R}_{1}$ é única. Mostremos que $\mathcal{R}_{1}$ é essencial, isto é, se $\left\{h_{t}\right\}$ é uma $G$-homotopia começando em $f_{1}$, deve existir uma $G$-classe de raízes $\mathcal{R}_{2}$ de $h_{1}$ tal que $\mathcal{R}_{1}\left\{h_{t}\right\}_{G} \mathcal{R}_{2}$.

Agora, vamos determinar $\mathcal{R}_{2}$ como segue. Como $\mathcal{R}$ é essencial, então existe uma G-classe de raízes $\mathcal{R}_{2}$ de $h_{1}$ tal que $\mathcal{R}\left(\left\{f_{t}\right\}\left\{h_{t}\right\}\right)_{G} \mathcal{R}_{2}$. Como $\mathcal{R}_{1}\left\{f_{t}^{-1}\right\}_{G} \mathcal{R}$, segue que $\mathcal{R}_{1}\left(f_{t}^{-1}\left\{f_{t}\right\}\left\{h_{t}\right\}\right)_{G} \mathcal{R}_{2}$. Portanto, $\mathcal{R}_{1}\left\{h_{t}\right\}_{G} \mathcal{R}_{2}$.

Note que $N_{G}(f, a) \leq M R_{G}[f, a]=\min \left\{\# \mathrm{k}^{-1}(\mathrm{a}) \mid \mathrm{k} \in[f]\right\}$, onde $k$ é tomada dentre todas as $G$-aplicações $G$-homotópicas a $f$.

OBSERVAÇÃo 2.2.7. Se $G=\{e\}$, então $N_{G}(f, a)$ se reduz ao número de Nielsen de raízes $N(f, a)$ de [B1].

\subsection{Levantamento da $G$-ação em $Y$}

Sejam $x_{0} \in f^{-1}(a)$ e $\eta: \widehat{Y} \rightarrow Y$ uma aplicação de revestimento correspondendo ao subgrupo $f_{\#} \pi_{1}\left(X, x_{0}\right)$ em $\pi_{1}(Y, a)$. Seja $\hat{f}: X \rightarrow \widehat{Y}$ um levantamento de $f$ através de 
$\eta$, isto é, $f=\eta \hat{f}$. Denote por $\mathcal{D}(\eta)=\{\delta: \widehat{Y} \rightarrow \widehat{Y}$ homeomorfismo $\mid \eta \delta=\eta\}$ o grupo das transformações de revestimento de $\eta$.

Considere o grupo $\Gamma_{G}(\widehat{Y})=\{\hat{g} \in \operatorname{Homeo}(\widehat{Y}) \mid \eta \hat{g}=g \eta$, para algum $g \in G\}$, onde $g$ é visto como um homeomorfismo de $Y$ induzido pela $G$-ação em $Y$.

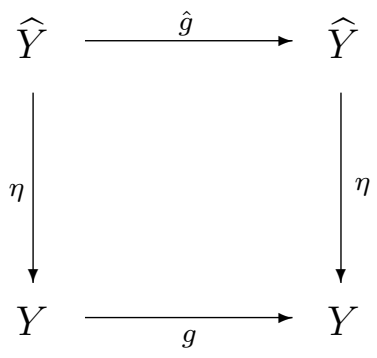

Assim obtemos a seguinte sequência exata

$$
1 \rightarrow \mathcal{D}(\eta) \stackrel{i}{\hookrightarrow} \Gamma_{G}(\widehat{Y}) \stackrel{p}{\rightarrow} G \rightarrow 1
$$

onde $i$ é a inclusão e $p(\hat{g})=g$ é a projeção.

A seguir, veremos que $f$ induz um homomorfismo de grupos $\varphi: G \rightarrow \Gamma_{G}(\widehat{Y})$. De fato, seja $x_{0} \in X$. Então existe um único levantamento $\varphi(g)$ de $g$ tal que $\varphi(g) \hat{f}\left(x_{0}\right)=$ $\hat{f}\left(g x_{0}\right)$. Como $\hat{f} g, \varphi(g) \hat{f}: X \rightarrow \widehat{Y}$ são levantamentos da mesma aplicação $f g=g f$ : $X \rightarrow Y$ e coincidem no ponto $x_{0}$, então pela unicidade da propriedade de levantamento, segue que $\hat{f} g=\varphi(g) \hat{f}$.

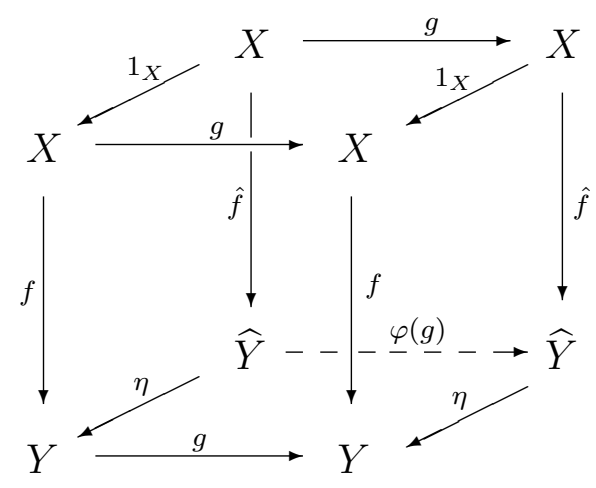

Observemos que a aplicação $\varphi$ aqui definida poderia ser indexada por $f$, mas essa indexação não é suficiente, como veremos nas páginas 24 e 29. A notação ideal seria $\varphi_{(f, \eta, \hat{f})}$, mas optamos por denotar tal aplicação apenas por $\varphi$. 
Lema 2.3.1. A aplicação $\varphi: G \rightarrow \Gamma_{G}(\widehat{Y})$ é um homomorfismo de grupos.

DEMONSTRAÇÃO. $\quad \varphi\left(g_{1} g_{2}\right) \hat{f}=\hat{f}\left(g_{1} g_{2}\right)=\left(\hat{f} g_{1}\right) g_{2}=\left(\varphi\left(g_{1}\right) \hat{f}\right) g_{2}=\varphi\left(g_{1}\right)\left(\hat{f} g_{2}\right)=$ $\varphi\left(g_{1}\right) \varphi\left(g_{2}\right) \hat{f}$, para quaisquer $g_{1}, g_{2} \in G$. Como $\varphi\left(g_{1} g_{2}\right)$ e $\varphi\left(g_{1}\right) \varphi\left(g_{2}\right)$ são levantamentos de $g_{1} g_{2}$, então pelo discutido acima, $\varphi\left(g_{1} g_{2}\right)=\varphi\left(g_{1}\right) \varphi\left(g_{2}\right)$.

Note que $p \varphi(g)=g$, para todo $g \in G$, logo a sequência (2.1) cinde. Então $\varphi$ é injetora e $\Gamma_{G}(\widehat{Y}) \simeq \mathcal{D}(\eta) \rtimes G$, isto é, $\Gamma_{G}(\widehat{Y})$ é o produto semi-direto de $\mathcal{D}(\eta)$ e $G$.

Vamos, agora, definir uma $G$-ação em $\widehat{Y}$ e provar que $\hat{f}: X \rightarrow \widehat{Y}$ e $\eta: \widehat{Y} \rightarrow Y$ são aplicações equivariantes.

Definição 2.3.2. Para todo $g \in G$ e $\hat{y} \in \widehat{Y}$, defina uma $G$-ação em $\widehat{Y}$ por

$$
\begin{aligned}
G \times \widehat{Y} & \longrightarrow \widehat{Y} \\
(g, \hat{y}) & \longmapsto g \cdot \hat{y}=\varphi(g)(\hat{y})
\end{aligned}
$$

Lema 2.3.3. As aplicações $\hat{f}: X \rightarrow \widehat{Y}$ e $\eta: \widehat{Y} \rightarrow Y$ são equivariantes.

DemonstraÇÃo. Com efeito, para todo $g \in G, x \in X$ e $\hat{y} \in \widehat{Y}$, temos que $\hat{f}(g x)=$ $\hat{f} g(x)=\varphi(g) \hat{f}(x)=g \cdot \hat{f}(x) \quad$ e $\quad \eta(g \cdot \hat{y})=\eta(\varphi(g) \hat{y})=g \eta(\hat{y})$.

Para o que faremos em seguida, precisaremos mostrar que $\eta$ é uma $G$-fibração. Para isso, considere o seguinte diagrama de $G$-espaços e $G$-aplicações.

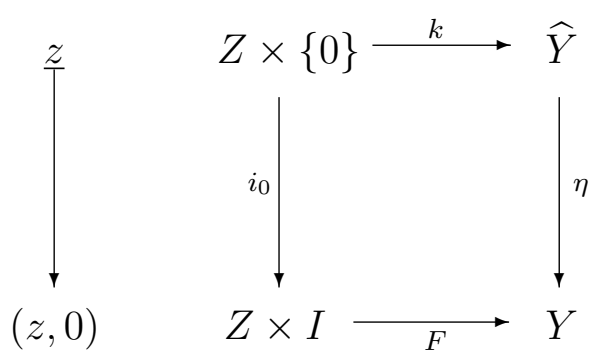

Como $\eta$ é uma fibração, pois é uma aplicação de revestimento, existe uma homotopia $\bar{F}: Z \times I \rightarrow \widehat{Y}$ de $k$ tal que $\eta \bar{F}=F$. Note que $g \bar{F}, \bar{F} g: Z \times I \rightarrow \widehat{Y}$ são levantamentos da mesma aplicação $g F=F g$ e coincidem em todos os pontos de $Z \times\{0\}$. Logo, $g \bar{F}=\bar{F} g$, ou seja, $\bar{F}$ é uma $G$-aplicação e, portanto $\eta$ é uma $G$-fibração. 
Teorema 2.3.4. Se $f^{\prime}: X \rightarrow Y$ é G-homotópica a $f$, então estas aplicações induzem a mesma G-ação no espaço de revestimento de Hopf $\widehat{Y}$ de $Y$.

Demonstração. Primeiramente, fixe em $\widehat{Y}$ a $G$-ação dada pela aplicação $\varphi$ (induzida por $f, \eta$ e $\hat{f}$ ) como na definição (2.3.2). Seja $\hat{f}^{\prime}: X \rightarrow \widehat{Y}$ um levantamento de $f^{\prime}$ através de $\eta: \widehat{Y} \rightarrow Y$. Como $\eta$ é uma $G$-fibração, então os levantamentos $\hat{f}^{\prime}$ e $\hat{f}$ de $f^{\prime}$ e $f$, respectivamente, são $G$-aplicações $G$-homotópicas.

Observe que, $f^{\prime}$ também induz um homomorfismo de grupos $\varphi^{\prime}: G \rightarrow \Gamma_{G}(\widehat{Y})$ tal que, para cada $g \in G, \varphi^{\prime}(g)$ é o único levantamento de $g$ tal que $\hat{f}^{\prime} g=\varphi^{\prime}(g) \hat{f}^{\prime}$ como levantamento de $f^{\prime} g=g f^{\prime}$ através de $\eta$. Logo, segue da definição (2.3.2), que $g * \hat{y}=\varphi^{\prime}(g) \hat{y}$ também é uma $G$-ação em $\widehat{Y}$. Além disso, pelo lema (2.3.3), $\hat{f}^{\prime}$ e $\eta$ são $G$-aplicações com esta nova ação $*$ em $\widehat{Y}$.

Note que, a aplicação $\hat{f}^{\prime}: X \rightarrow \widehat{Y}$ é equivariante com ambas as $G$-ações $\cdot$ e * em $\widehat{Y}$, induzidas, respectivamente, por $\varphi$ e $\varphi^{\prime}$. Então $g * \hat{f}^{\prime}(x)=\varphi^{\prime}(g) \hat{f}^{\prime}(x)=\hat{f}^{\prime} g(x)=$ $g \cdot \hat{f}^{\prime}(x)=\varphi(g) \hat{f}^{\prime}(x)$, para todo $g \in G, x \in X$. Logo, $\varphi^{\prime}(g) \hat{f}^{\prime}(x)=\varphi(g) \hat{f}^{\prime}(x)$, para todo $g \in G, x \in X$. Então, para todo $g \in G$, temos que $\varphi^{\prime}(g)=\varphi(g)$, pois as aplicações $\varphi^{\prime}(g), \varphi(g): \widehat{Y} \rightarrow \widehat{Y}$ são levantamentos da mesma aplicação $g: Y \rightarrow Y$ e coincidem em todos os pontos de $\hat{f}^{\prime}(X)$. Portanto, as $G$-ações $*$ e $\cdot$, induzidas por $\varphi^{\prime}$ e $\varphi$, respectivamente, coincidem. Ou seja, $\varphi^{\prime}=\varphi: G \rightarrow \Gamma_{G}(\widehat{Y})$.

Mais especificamente, o teorema acima mostra que todas as aplicações na classe de $G$-homotopia da $f$ induzem a mesma $G$-ação no espaço de revestimento de Hopf $\widehat{Y}$ de $Y$.

O próximo passo é estabelecer uma versão equivariante do teorema (1.4.5) e, assim, caracterizar as $G$-classes de raízes de $f$ em termos de um levantamento de Hopf desta aplicação.

Proposição 2.3.5. Sejam $f: X \rightarrow Y$ uma $G$-aplicação e $a \in Y^{G} \neq \emptyset$. Suponha $\left\{f_{t}: X \rightarrow Y\right\}$ uma G-homotopia começando em $f$ e seja $\left\{\hat{f}_{t}: X \rightarrow \widehat{Y}\right\}$ um levantamento de $\left\{f_{t}\right\}$ através de $\eta: \widehat{Y} \rightarrow Y$. Então 
(1) As raízes $x_{0}$ e $x_{1}$ de $f$ em a são $G$-Nielsen equivalentes se, e somente se, $\hat{f}\left(x_{0}\right)=$ $g \cdot \hat{f}\left(x_{1}\right)$, para algum $g \in G$.

(2) Uma raiz $x_{0} \in f^{-1}(a)$ está $\left\{f_{t}\right\}_{G}$-relacionada a uma raiz $x_{1}$ de $f_{1}$ em a se, e somente se, $\hat{f}\left(x_{0}\right)=g \cdot \hat{f}_{1}\left(x_{1}\right)$, para algum $g \in G$.

DemonstraçÃo. $(1)(\Rightarrow)$ Primeiro, suponha que $x_{0} \sim_{G} x_{1}$. Então existem duas possibilidades.

(i) Se $x_{0}=g x_{1}$, para algum $g \in G$, então $\hat{f}\left(x_{0}\right)=\hat{f}\left(g x_{1}\right)=\varphi(g) \hat{f}\left(x_{1}\right)=g \cdot \hat{f}\left(x_{1}\right)$.

(ii) Se existe um caminho $c: I \rightarrow X$ de $x_{0}$ para $g x_{1}$, para algum $g \in G$ tal que $f c \simeq \bar{a} \operatorname{rel}\{0,1\}$, então $x_{0} \sim g x_{1}$. Logo, pelo teorema (1.4.5), $\hat{f}\left(x_{0}\right)=\hat{f}\left(g x_{1}\right) ;$ conseqüentemente, temos que $\hat{f}\left(x_{0}\right)=g \cdot \hat{f}\left(x_{1}\right)$.

$(\Leftarrow)$ Agora, suponha que $\hat{f}\left(x_{0}\right)=g \cdot \hat{f}\left(x_{1}\right)$, para algum $g \in G$. Então $\hat{f}\left(x_{0}\right)=$ $\varphi(g) \hat{f}\left(x_{1}\right)=\hat{f}\left(g x_{1}\right)$. Logo, segue do teorema (1.4.5) que $x_{0}$ e $g x_{1}$ pertencem a mesma classe de raízes de $f$ e, portanto, estão na mesma $G$-classe de raízes.

$(2)(\Rightarrow)$ Seja $x_{0}\left\{f_{t}\right\}_{G} x_{1}$. Então existe um caminho $c: I \rightarrow X$ de $x_{0}$ para $g x_{1}$, para algum $g \in G$ tal que $\left\{f_{t} \circ c(t)\right\} \simeq \bar{a} \operatorname{rel}\{0,1\}$. Logo, $x_{0}\left\{f_{t}\right\} g x_{1}$ e, pelo teorema (1.4.5), temos então que $\hat{f}\left(x_{0}\right)=\hat{f}_{1}\left(g x_{1}\right)=g \cdot \hat{f}_{1}\left(x_{1}\right)$.

$(\Leftarrow)$ Se $\hat{f}\left(x_{0}\right)=g \cdot \hat{f}_{1}\left(x_{1}\right)$, para algum $g \in G$, então $\hat{f}\left(x_{0}\right)=\hat{f}_{1}\left(g x_{1}\right)$, pois $\hat{f}_{1}$ é equivariante. Portanto, pelo teorema (1.4.5), segue que $x_{0}\left\{f_{t}\right\} g x_{1}$, o que implica que $x_{0}\left\{f_{t}\right\}_{G} x_{1}$.

Portanto, as $G$-classes de raízes de Nielsen são os conjuntos não-vazios da forma $\hat{f}^{-1}(G(\hat{y}))$, para cada $\hat{y} \in \eta^{-1}(a)$.

Corolário 2.3.6. Nas mesmas hipóteses da proposição (2.3.5), sejam $x_{0} \in f^{-1}(a)$ e $\mathcal{R}$ a $G$-classe de raízes contendo $x_{0}$. Então o número de classes de raízes contidas em $\mathcal{R}$ é exatamente a cardinalidade da órbita $G\left(\hat{f}\left(x_{0}\right)\right)$.

DemonstraçÃo. Segue imediatamente dos resultados (1.4.5) e (2.3.5). 
Corolário 2.3.7. Nas mesmas hipóteses da proposição (2.3.5), para qualquer $\hat{y} \in$ $\eta^{-1}(a)$, uma $G$-classe de raízes $\hat{f}^{-1}(G(\hat{y}))$ é essencial se, e somente se, $\hat{f}_{1}^{-1}(G(\hat{y})) \neq \emptyset$ para qualquer G-homotopia $\left\{\hat{f}_{t}\right\}$ começando em $\hat{f}$.

Já vimos que $N_{G}(f, a)$ é um invariante por $G$-homotopias em (2.2.6). No entanto, este resultado também pode ser obtido do corolário (2.3.7) acima.

Tendo em vista a definição (2.3.2) da $G$-ação em $\widehat{Y}$, é natural surgir a seguinte pergunta: fixado um levantamento $\hat{f}: X \rightarrow \widehat{Y}$ de $f$, qual ação em $\widehat{Y}$ torna uma transformação de revestimento $\delta: \widehat{Y} \rightarrow \widehat{Y}$ equivariante?

Para responder a essa pergunta, seja $\delta \in \mathcal{D}(\eta)$. Então $\delta \hat{f}: X \rightarrow \widehat{Y}$ é um levantamento de $f: X \rightarrow Y$ através de $\eta: \widehat{Y} \rightarrow Y$. Logo, $f$ induz um homomorfismo de grupos $\varphi_{\delta}: G \rightarrow \Gamma_{G}(\widehat{Y})$ tal que para cada $g \in G, \varphi_{\delta}(g)$ é o único levantamento de $g$ satisfazendo $\varphi_{\delta}(g) \delta \hat{f}=\delta \hat{f} g$ como levantamento de $g f=f g$ através de $\eta$.

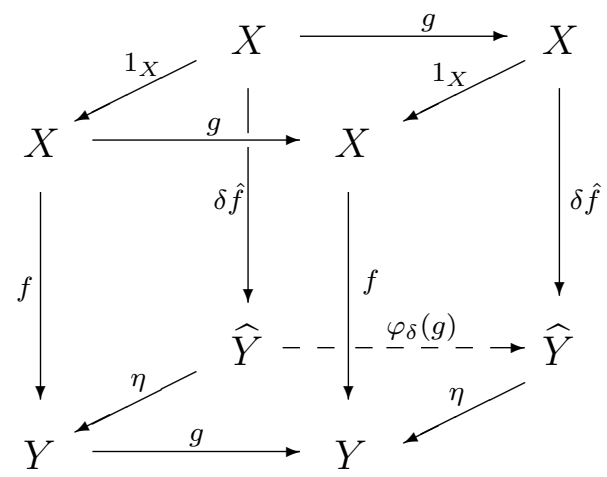

Defina a seguinte $G$-ação em $\widehat{Y}:(g, \hat{y}) \longmapsto g \circ_{\delta} \hat{y}=\varphi_{\delta}(g)(\hat{y})$. Como $\varphi_{\delta}(g) \delta \hat{f} \stackrel{(2.3)}{=} \delta \hat{f} g$, então $\delta^{-1} \varphi_{\delta}(g) \delta \hat{f}=\hat{f} g \stackrel{(2.2)}{=} \varphi(g) \hat{f}$, e assim, $\delta^{-1} \varphi_{\delta}(g) \delta=\varphi(g)$. Ou seja, as ações $\cdot$ e $o_{\delta}$, dadas por $\varphi$ e $\varphi_{\delta}$ respectivamente, são conjugadas por $\delta$. Logo, para todo $g \in G$ e $\hat{y} \in \widehat{Y}$, temos que $\delta(g \cdot(\hat{y}))=\delta \varphi(g)(\hat{y})=\varphi_{\delta}(g) \delta(\hat{y})=g \circ_{\delta} \delta(\hat{y})$. Portanto, com a notação utilizada acima, obtemos o seguinte resultado.

Proposição 2.3.8. As transformações de revestimento $\delta:(\widehat{Y}, \cdot) \rightarrow\left(\widehat{Y}, \circ_{\delta}\right)$ são equivariantes. 


\subsection{Relação entre classes e $G$-classes de raízes}

Retomando o discutido na seção 2.1, descreveremos com mais detalhes as $G$-classes de raízes. A exposição desta seção baseia-se na seção 2.2 de [Fa], onde foram estudadas $G$-classes de coincidências e classes de coincidências locais.

Proposição 2.4.1. Seja $\alpha$ uma classe de raizes contida em uma $G$-classe de raizes $\mathcal{R}$ e seja $x_{0} \in \alpha$. Se $\beta$ é outra classe de raizes em $\mathcal{R}$, disjunta de $\alpha$, então $\beta=g \alpha$, para algum $g \in G$.

Demonstração. Para todo $g \in G$, mostremos que ga é uma classe de raízes contida em $\mathcal{R}$. De fato, Sejam $y_{0}, y_{1} \in g \alpha$. Então existem $x_{0}, x_{1} \in \alpha$ tais que $y_{0}=g x_{0}$ e $y_{1}=g x_{1}$. Como $x_{0}, x_{1} \in \alpha$, existe um caminho $c: I \rightarrow X$ de $x_{0}$ para $x_{1}$ satisfazendo $f c \simeq \bar{a} \operatorname{rel}\{0,1\}$. Considere o caminho $d=g c: I \rightarrow X$ de $g x_{0}=y_{0}$ para $g x_{1}=y_{1}$. Logo, $f d \simeq \bar{a}$ rel $\{0,1\}$. Assim, $g \alpha$ está contido em uma classe de raízes de $f$, digamos $\gamma$. Seja, agora, $z \in \gamma$. Mostremos que $z \in g \alpha$. Como $g \alpha \subset \gamma$, existem $x \in g \alpha$ e um caminho $k: I \rightarrow X$ de $x$ para $z$ tal que $f k \simeq \bar{a} \operatorname{rel}\{0,1\}$. Como $x \in g \alpha$, então existe $y \in \alpha$ tal que $x=g y$. Considere o caminho $g^{-1} k: I \rightarrow X$. Logo, $g^{-1} k(0)=g^{-1} x=y \in \alpha, g^{-1} k(1)=g^{-1} z$ e $f g^{-1} k \simeq \bar{a} \operatorname{rel}\{0,1\}$. Portanto, $g^{-1} z \in \alpha$, ou seja, $z \in g \alpha$. Então $g \alpha$ é uma classe de raízes. Agora, note que, $x_{0} \in \alpha \subset \mathcal{R}$, $g x_{0} \in g \alpha$ e os pontos $x_{0}$ e $g x_{0}$ estão na mesma órbita, então segue da definição (2.1.1) que $g \alpha \subset \mathcal{R}$.

Suponha $\alpha$ e $\beta$ classes de raízes disjuntas em $\mathcal{R}$. Se tal classe $\beta$ não existir, então $\alpha=\mathcal{R}$.

Seja $x_{1} \in \beta$. Então, pelo teorema (1.4.5), $\hat{f}\left(x_{0}\right) \neq \hat{f}\left(x_{1}\right)$. Entretanto, a proposição (2.3.5) nos diz que $\hat{f}\left(x_{0}\right)$ e $\hat{f}\left(x_{1}\right)$ pertencem à mesma órbita, ou seja, existe $g \in G$ tal que $\hat{f}\left(x_{1}\right)=g \cdot \hat{f}\left(x_{0}\right)=\hat{f}\left(g x_{0}\right)$. Logo, $x_{1}$ e $g x_{0}$ pertencem à mesma classe de raízes. Portanto, $\beta=g \alpha$.

Agora, considere o conjunto $G_{\alpha}^{x_{0}}=\left\{g \in G \mid g x_{0} \in \alpha\right\}$. Pode-se mostrar (o que não o faremos aqui) que $G_{\alpha}^{x_{0}}$ é um subgrupo de $G$. 
Lema 2.4.2. $G_{\alpha}^{x_{0}}$ independe do ponto $x_{0} \in \alpha$ escolhido.

DemonstraçÃo. Seja $x_{1} \neq x_{0} \in \alpha$. Mostremos que $G_{\alpha}^{x_{0}}=G_{\alpha}^{x_{1}}$. Seja $g \in G_{\alpha}^{x_{0}}$, então $g x_{0} \in \alpha$. Como $g x_{0}, x_{1} \in \alpha$, existe um caminho $c: I \rightarrow X$ de $g x_{0}$ para $x_{1}$ tal que $f c \simeq \bar{a} \operatorname{rel}\{0,1\}$. Além disso, $x_{0}, x_{1} \in \alpha$, então existe um caminho $d: I \rightarrow X$ tal que $d(0)=x_{0}, d(1)=x_{1}$ e $f d \simeq \bar{a} \operatorname{rel}\{0,1\}$.

Seja $g d^{-1} * c: I \rightarrow X$. Logo, $g d^{-1} * c(0)=g x_{1}, g d^{-1} * c(1)=x_{1} \in \alpha$ e $f\left(g d^{-1} * c\right) \simeq \bar{a}$ $\operatorname{rel}\{0,1\}$. Então $g x_{1} \in \alpha$, ou seja, $g \in G_{\alpha}^{x_{1}}$, e portanto, $G_{\alpha}^{x_{0}} \subset G_{\alpha}^{x_{1}}$. Analogamente prova-se que $G_{\alpha}^{x_{1}} \subset G_{\alpha}^{x_{0}}$.

Em vista do lema anterior, passaremos a denotar $G_{\alpha}^{x_{0}}$ por $G_{\alpha}$.

Lema 2.4.3. Sejam $\alpha$ e ga classes de raízes contidas na mesma $G$-classe de raízes $\mathcal{R}$. Então $G_{\alpha}$ e $G_{g \alpha}$ são conjugados.

DemonstraÇÃo. Sejam $x_{0} \in \alpha$ e $g^{\prime} \in G_{g \alpha}$. Então $g^{\prime} g x_{0} \in g \alpha$. Logo, existe um caminho $c: I \rightarrow X$ de $g x_{0}$ para $g^{\prime} g x_{0}$ tal que $f c \simeq \bar{a}$ rel $\{0,1\}$. Considere o caminho $d=g^{-1} c: I \rightarrow X$. Então $d(0)=x_{0} \in \alpha, d(1)=g^{-1} g^{\prime} g x_{0}$ e $f d \simeq \bar{a} \operatorname{rel}\{0,1\}$. Logo, $g^{-1} g^{\prime} g x_{0} \in \alpha$ e assim, $g^{-1} g^{\prime} g \in G_{\alpha}$. Portanto, $g^{-1} G_{g \alpha} g \subset G_{\alpha}$. De modo análogo prova-se a inclusão contrária.

Considere o conjunto das classes laterais $G / G_{\alpha}=\left\{H_{1}, H_{2}, \ldots, H_{i}, \ldots\right\}$, onde $H_{1}=$ $G_{\alpha}$. Denote por $h_{i}$ um elemento de $H_{i}, i=1,2, \ldots$.

Lema 2.4.4. A classe de raizes $h_{i} \alpha$ independe da escolha do elemento $h_{i} \in H_{i}$, $i \in\{1,2, \ldots\}$.

DemonstraÇÃo. Sejam $h_{1}$ e $h_{2} \in H_{i}=g_{i} G_{\alpha}$, para algum $i \in\{1,2, \ldots\}$, onde $g_{i} \in G$. Logo, $h_{1}=g_{i} \bar{g}$ e $h_{2}=g_{i} \overline{\bar{g}} \operatorname{com} \bar{g}$ e $\overline{\bar{g}} \in G_{\alpha}$. Então $h_{1} \alpha=g_{i} \bar{g} \alpha=g_{i} \alpha=g_{i} \overline{\bar{g}}=h_{2} \alpha$.

Lema 2.4.5. Seja $\mathcal{R}$ a $G$-classe de raízes contendo a classe de Nielsen de raízes $\alpha$ e seja $x_{0} \in \alpha$. Então $\mathcal{R}=\bigcup_{i} h_{i} \alpha$. 
Demonstração. Primeiro, mostremos que $\mathcal{R} \subset \bigcup_{i} h_{i} \alpha$. Seja $y \in \mathcal{R}$. Então temos duas possibilidades.

(i) $y=g x_{0}$, para algum $g \in G$. Neste caso, seja $H_{i}$ a classe de $G / G_{\alpha}$ contendo $g$. Logo, $y \in g \alpha$.

(ii) Existe um caminho $c: I \rightarrow X$ de $y$ para $g^{\prime} x_{0}$, para algum $g^{\prime} \in G$ tal que $f c \simeq \bar{a}$ $\operatorname{rel}\{0,1\}$. Então $y \in g^{\prime} \alpha$, pois $g^{\prime} \in H_{i}$ para algum $i$.

Observe que a inclusão $\bigcup_{i} h_{i} \alpha \subset \mathcal{R}$ decorre da proposição (2.4.1) e da definição (2.1.1). Note ainda que, pela definição (1.4.1), esta reunião é disjunta.

Finalmente, vamos relacionar os resultados acima e a $G$-ação definida no espaço de revestimento $\widehat{Y}$ (vide definição 2.3.2).

Proposição 2.4.6. Seja $G$ um grupo cíclico de ordem prima. Então $G_{\hat{y}}=G$ ou $G_{\hat{y}}=\{e\}$, para qualquer $\hat{y} \in \eta^{-1}(a) \cap \hat{f}(X)$.

Demonstração. Como $G$ é um grupo cíclico de ordem prima, então $G$ não possui subgrupos próprios. Portanto, para qualquer classe de raízes $\alpha$ de $f, G_{\alpha}=G$ ou $G_{\alpha}=\{e\}$. Seja $x_{0} \in \alpha \subset \mathcal{R}$. Se $G_{\alpha}=G$, então $g \alpha=\alpha=\mathcal{R}$, para todo $g \in G$. Logo, pelo corolário (2.3.6), \#G( $\left.\hat{f}\left(x_{0}\right)\right)=1$, ou seja, $G\left(\hat{f}\left(x_{0}\right)\right)=\hat{f}\left(x_{0}\right)$. Então, $G_{\hat{f}\left(x_{0}\right)}=G$. Mas se $G_{\alpha}=\{e\}$, então para todo $g \neq e \in G$, as classes de raízes $\alpha$ e $g \alpha$ são disjuntas. Assim, a cardinalidade da órbita $G\left(\hat{f}\left(x_{0}\right)\right)$ é exatamente a cardinalidade de $G$, pelo corolário (2.3.6). Portanto, $G_{\hat{f}\left(x_{0}\right)}=\{e\}$.

\subsection{O G-número de Reidemeister de raízes}

Nosso primeiro objetivo, nesta seção, é definir o $G$-número de Reidemiester $R_{G}(f, a)$ usando, como principal ferramenta, o revestimento de Hopf $\eta: \widehat{Y} \rightarrow Y$ para $f: X \rightarrow Y$. Para encerrarmos esta seção, veremos alguns exemplos dos números $R(f, a)$ e $R_{G}(f, a)$ e provaremos que $R_{G}(f, a)$ é um limitante superior para $N_{G}(f, a)$. 
Considere a restrição da $G$-ação, definida em $\widehat{Y}$, ao conjunto $\eta^{-1}(a)$. Como $\eta$ é equivariante e $a \in Y^{G} \neq \emptyset$, então $\eta^{-1}(a)$ é um $G$-conjunto. Lembremos que, pela proposição (2.3.5), o conjunto das $G$-classes de raízes está em correspondência com um subconjunto de $G$-órbitas $G(\hat{y})$ em $\eta^{-1}(a)$. Assim, o número de tais $G$-órbitas é, portanto, um limitante superior para o número de $G$-classes de raízes essenciais de $f$ em $a$. Sendo assim, temos a seguinte definição.

Definição 2.5.1. O G-número de Reidemeister de $f$ é o número de G-órbitas $G(\hat{y})$, onde $\hat{y} \in \eta^{-1}(a)$.

Convém observarmos que $R_{G}(f, a)$ não depende da escolha do ponto $a$, mas ainda precisamos mostrar que $R_{G}(f, a)$ independe da escolha do revestimento de Hopf para $f$. Para isso, seja $\tilde{\eta}: \tilde{Y} \rightarrow Y$ outra aplicação de revestimento associado ao subgrupo $f_{\#} \pi_{1}\left(X, x_{0}\right) \subseteq \pi_{1}(Y, a)$, onde $x_{0} \in f^{-1}(a)$. Seja ainda $\tilde{f}: X \rightarrow \tilde{Y}$ um levantamento de $f$ relativamente a $\tilde{\eta}$ (isto é, $f=\tilde{\eta} \tilde{f})$. Então $\tilde{\eta}_{\#} \pi_{1}\left(\tilde{Y}, \tilde{f}\left(x_{0}\right)\right)=f_{\#} \pi_{1}\left(X, x_{0}\right)=$ $\eta_{\#} \pi_{1}\left(\widehat{Y}, \hat{f}\left(x_{0}\right)\right)$, e portanto, existe um homeomorfismo $\psi: \widehat{Y} \rightarrow \tilde{Y}$ tal que $\tilde{\eta} \psi=\eta$ e $\psi\left(\hat{f}\left(x_{0}\right)\right)=\tilde{f}\left(x_{0}\right)$.

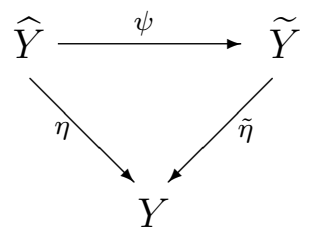

Note que, a aplicação $\eta$ induz um homomorfismo de grupos $\bar{\varphi}: G \rightarrow \Gamma_{G}(\widetilde{Y})$ tal que, para cada $g \in G, \bar{\varphi}(g)$ é o único levantamento de $g$ por $\tilde{\eta}$ (isto é, $\tilde{\eta} \bar{\varphi}(g)=g \tilde{\eta}$ ) tal que $\bar{\varphi}(g) \psi=\psi \varphi(g)$ como levantamento de $g \eta=\eta \varphi(g)$. Assim sendo, $\widetilde{Y}$ torna-se um $G$-espaço com a ação $g \circ \tilde{y}:=\bar{\varphi}(g)(\tilde{y})$, para todo $\tilde{y} \in \tilde{Y}, g \in G$. Então, $\psi: \widehat{Y} \rightarrow \widetilde{Y}$ e $\tilde{\eta}: \tilde{Y} \rightarrow Y$ serão $G$-aplicações. 


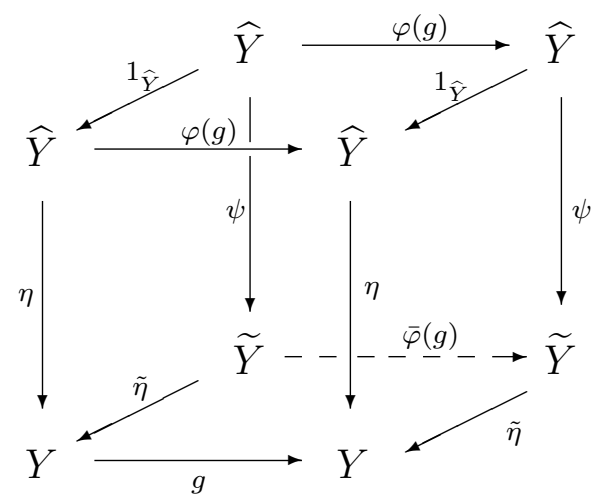

No entanto, a aplicação $f: X \rightarrow Y$ também induz um homomorfismo de grupos $\tilde{\varphi}: G \rightarrow \Gamma_{G}(\tilde{Y})$ tal que, para cada $g \in G$, existe um único levantamento $\tilde{\varphi}(g)$ de $g$ através de $\tilde{\eta}$ (ou seja, $\tilde{\eta} \tilde{\varphi}(g)=g \tilde{\eta}$ ) satisfazendo $\tilde{\varphi}(g) \tilde{f}=\tilde{f} g$ como levantamento de $g f=f g$. Portanto, podemos definir outra $G$-ação em $\widetilde{Y}$, que será denotada por $g * \tilde{y}:=\tilde{\varphi}(g)(\tilde{y})$, para todo $\tilde{y} \in \tilde{Y}, g \in G$. Logo, $\tilde{f}: X \rightarrow \tilde{Y}$ e $\tilde{\eta}: \tilde{Y} \rightarrow Y$ tornam-se G-aplicações.

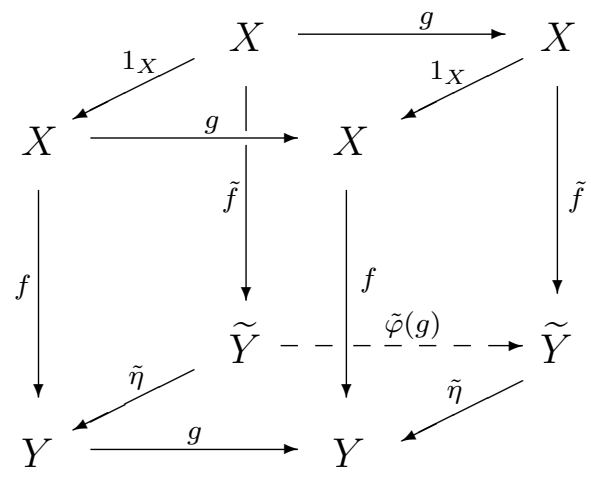

Tendo em vista os conceitos apresentados acima, vamos mostrar que o número de órbitas em $\eta^{-1}(a)$, com a $G$-ação dada por $\varphi$, é o mesmo do que o número de órbitas em $\tilde{\eta}^{-1}(a)$, com a ação dada por $\tilde{\varphi}$. É importante observar que ambos os homomorfismos $\varphi$ e $\tilde{\varphi}$ são induzidos por $f$ e, além disso, $\psi \varphi(g) \hat{f} \stackrel{(2.2)}{=} \psi \hat{f} g=\tilde{f} g \stackrel{(2.5)}{=} \tilde{\varphi}(g) \tilde{f}=\tilde{\varphi}(g) \psi \hat{f}$. Então $\psi \varphi(g)=\tilde{\varphi}(g) \psi$, ou seja, $\varphi(g)=\psi \tilde{\varphi}(g) \psi^{-1}$.

Como $\psi: \widehat{Y} \rightarrow \widetilde{Y}$ é um homeomorfismo, a condição $\tilde{\eta} \psi=\eta$ significa que $\psi$ induz, para cada $y \in Y$, uma bijeção da fibra $\eta^{-1}(y)$ sobre a fibra $\tilde{\eta}^{-1}(y)$; logo, tais fibras têm a mesma cardinalidade. Além disso, como $\psi$ é equivariante, dado $\hat{y} \in \eta^{-1}(y)$ arbitrário, existe uma bijeção entre as órbitas $G(\hat{y})=\{g \cdot \hat{y} \mid g \in G\}=\{\varphi(g)(\hat{y}) \mid g \in G\}$ e 
$G(\psi(\hat{y}))=\{g \circ \psi(\hat{y}) \mid g \in G\}=\{\bar{\varphi}(g) \psi(\hat{y}) \mid g \in G\}=\{\psi \varphi(g)(\hat{y}) \mid g \in G\}$. Portanto, o número de órbitas em $\eta^{-1}(a)$, com a ação $\cdot$ dada por $\varphi$, é o mesmo do que o número de órbitas em $\tilde{\eta}^{-1}(a)$, com a ação o dada por $\bar{\varphi}$.

Agora, note que, $\bar{\varphi}(g) \tilde{f}=\bar{\varphi}(g) \psi \hat{f} \stackrel{(2.4)}{=} \psi \varphi(g) \hat{f} \stackrel{(2.2)}{=} \psi \hat{f} g=\tilde{f} g \stackrel{(2.5)}{=} \tilde{\varphi}(g) \tilde{f}$. Então $\bar{\varphi}(g)=\tilde{\varphi}(g): \tilde{Y} \rightarrow \tilde{Y}$, pois ambos são levantamentos da aplicação $g: Y \rightarrow Y$ e coincidem em $\tilde{f}(X)$. Portanto, as ações $\circ$ e $*$, induzidas respectivamente por $\bar{\varphi}$ e $\tilde{\varphi}$, coincidem. Portanto, o $G$-número de Reidemeister $R_{G}(f, a)$ está bem definido, conforme queríamos demonstrar.

Como conseqüência da teoria desenvolvida neste capítulo, obtemos o seguinte resultado.

Teorema 2.5.2. Sejam $f: X \rightarrow Y$ uma G-aplicação e $a \in Y^{G} \neq \emptyset$. Considere um revestimento de Hopf $\eta: \widehat{Y} \rightarrow Y$ para $f$ e seja $\hat{f}: X \rightarrow \widehat{Y}$ um levantamento de Hopf de $f$ por $\eta$. Entãa

(1) $f$ tem no máximo $R_{G}(f, a) G$-classes de Nielsen de raízes.

(2) $N_{G}(f, a) \leq R_{G}(f, a)$.

(4) $R_{G}(f, a) \leq R(f, a)$.

(4) Se G é finito e $R(f, a)=\infty$, então $R_{G}(f, a)=\infty$.

OBSERVAÇão 2.5.3. Se $G=\{e\}$, então $R_{G}(f, a)$ se reduz ao número de Reidemeister $R(f, a)$ de [B1].

Antes de encerrarmos esta seção, convém apresentarmos alguns exemplos que ilustrem os cálculos dos números $R(f, a)$ e $R_{G}(f, a)$.

Exemplo 2.5.4. Considere a ação de $\mathbb{Z}_{2} \simeq\{-1,1\}$ em $S^{1}$ dada por $-1 \cdot e^{\theta i}=e^{-\theta i}$. Sejam $f: S^{1} \rightarrow S^{1}$ a $\mathbb{Z}_{2}$-aplicação definida por $f\left(e^{\theta i}\right)=e^{-\theta i}$ e $a=-1$. A aplicação 
de revestimento correspondendo ao subgrupo $f_{\#} \pi_{1}\left(S^{1}\right) \simeq \mathbb{Z}$ é a aplicação identidade $i d: S^{1} \rightarrow S^{1}$. Então $R(f, a)=R_{\mathbb{Z}_{2}}(f, a)=1$.

Exemplo 2.5.5. No exemplo (2.2.4) foi dada a $S^{1}$-aplicação $f: S^{3} \rightarrow S^{2}=S^{3} / S^{1}$ constante em $a=[e]$, a classe do elemento identidade $e$ no grupo de Lie compacto conexo $S^{3}$. Como $S^{2}$ é simplemente conexo, todo espaço de revestimento sobre $S^{2}$ é equivalente ao espaço de revestimento trivial, isto é, $i d: S^{2} \rightarrow S^{2}$. Logo, $R(f, a)=$ $R_{\mathbb{Z}_{2}}(f, a)=1$.

Exemplo 2.5.6. Usaremos a mesma aplicação dada no exemplo (2.2.5). Então, seja $f: S^{2} \rightarrow \mathbb{R} P^{2}$ a projeção natural e considere a ação antípoda de $\mathbb{Z}_{2} \simeq\{-1,1\}$ em $S^{2}$ e trivial em $\mathbb{R} P^{2}$. Como $S^{2}$ é simplesmente conexo, então $S^{2}$ é o espaço de revestimento universal sobre $\mathbb{R} P^{2}$ relativo a $f$. Logo, $R(f)=2$.

Para calcularmos $R_{\mathbb{Z}_{2}}(f)$, precisamos definir o homomorfismo $\varphi: \mathbb{Z}_{2} \rightarrow \Gamma_{\mathbb{Z}_{2}}\left(S^{2}\right)$ que induz a $\mathbb{Z}_{2}$-ação no revestimento $S^{2}$. Vimos na seção 2.3 (vide páginas 19 e 20 ) que $\varphi$ é injetora e o grupo $\Gamma_{\mathbb{Z}_{2}}\left(S^{2}\right)$ é definido por

$$
\Gamma_{\mathbb{Z}_{2}}\left(S^{2}\right)=\left\{\hat{g} \in \operatorname{Homeo}\left(S^{2}\right) \mid f \hat{g}=g f \text { para algum } g \in \mathbb{Z}_{2}\right\}
$$

Além disso, $\Gamma_{\mathbb{Z}_{2}}\left(S^{2}\right) \cong \mathcal{D}(f) \rtimes \mathbb{Z}_{2} \cong \mathbb{Z}_{2} \rtimes \mathbb{Z}_{2}$. Lembremos que para cada $g \in \mathbb{Z}_{2}, \varphi(g)$ é o único levantamento de $g$ tal que $\varphi(g) \hat{f}=\hat{f} g$ como levantamento de $f g=g f$, onde $\hat{f}$ denota um levantamento de $f$.

Observe que, as aplicações identidade $i d: S^{2} \rightarrow S^{2}$ e antípoda $A: S^{2} \rightarrow S^{2}$ são levantamentos de $f$ (relativamente a $f$ ). Primeiramente, considere $i d$ como levantamento de $f$. Então $\varphi(g)=g$, ou seja, para todo $x \in S^{2}$, segue que $\varphi(1)(x)=x$ e $\varphi(-1)(x)=-x$.

Agora, seja $A$ o levantamento de $f$. Logo, $\varphi(g) A=A g$, e portanto, para todo $x \in S^{2}$ temos que $\varphi(1)(-x)=-x$ e $\varphi(-1)(-x)=x$.

Como $\varphi$ é injetora, segue que $\mathbb{Z}_{2}$ também atua antipodalmente em $S^{2}$. Portanto, $R_{\mathbb{Z}_{2}}(f)=1$. 
Exemplo 2.5.7. Sejam $X=Y=S^{1} \times S^{1} \times S^{1}$ e $G=\mathbb{Z}_{3}=\langle\xi\rangle$. Considere a

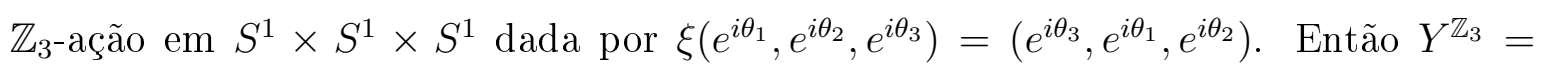
$\left\{\left(e^{i \theta}, e^{i \theta}, e^{i \theta}\right) \mid e^{i \theta} \in S^{1}\right\}$. Seja $a \in Y^{\mathbb{Z}_{3}}$.

Seja $f: S^{1} \times S^{1} \times S^{1} \rightarrow S^{1} \times S^{1} \times S^{1}$ a aplicação constante em $a$. Logo, $f$ é $\mathbb{Z}_{3^{-}}$ equivariante. Note que, como $f$ é constante em $a$, então o revestimento associado ao subgrupo $f_{\#} \pi_{1}\left(S^{1} \times S^{1} \times S^{1}\right)$ é o revestimento universal $\eta: \mathbb{R} \times \mathbb{R} \times \mathbb{R} \rightarrow S^{1} \times S^{1} \times S^{1}$. Portanto, $R(f, a)=\eta^{-1}(a)=\infty$ e pelo teorema 2.5.2(4), segue que $R_{\mathbb{Z}_{3}}(f, a)=\infty$. 


\section{Capítulo 3}

\section{Propriedade Wecken Equivariante}

O primeiro a trabalhar com a realização do número de Nielsen (de ponto-fixo) foi Franz Wecken [We], que em 1941-2 publicou uma série de artigos atacando este problema. Por essa razão, este capítulo leva seu nome.

Usando a teoria desenvolvida no capítulo 2, mostraremos alguns resultados da realização do número de Nielsen equivariante de raízes, quando este é zero.

Uma importante motivação para este trabalho foi o Teorema 3 de [W4], no qual P. Wong mostrou que se uma $G$-aplicação $f: X \rightarrow Y$ entre $G$-variedades diferenciáveis fechadas orientáveis e de mesma dimensão tem grau zero, isto é, $\operatorname{deg} f=0$, então $N_{G}(f, a)=0$ (sob algumas hipóteses apropriadas sobre a $G$-ação em $X$ e em $Y$ ). A diferença crucial entre o Teorema 3 de [W4] e os resultados apresentados neste capítulo, é que, $X$ será considerado como um $G$-espaço topológico e $Y$ uma $G$-varidade topológica. Conforme mencionamos na introdução deste trabalho, a propriedade Wecken para raízes não tem sido muito estudada em situações onde os espaços não são variedades ou são variedades de dimensões diferentes. 


\section{$3.1 \quad G$-ANR e $G$-ANE}

Sejam $G$ um grupo finito e $M$ um $G$-espaço topológico. Considere uma órbita $G(x)$ em $M$, onde $x \in M$ e, seja $V$ um espaço vetorial euclidiano no qual $G_{x}$ opera ortogonalmente. Então um tubo linear sobre $G(x)$ em $M$ é um $G$-mergulho topológico sobre uma vizinhança aberta de $G(x)$ da forma $G \times_{G_{x}} V \rightarrow M$. Então um $G$-tubo sobre $G(x)$ pode ser visto como um par $T=(T, r)$, onde $T$ é uma vizinhança aberta invariante de $G(x)$ e $r: T \rightarrow G(x)$ é uma $G$-retração. (Vide [M], p.483).

Dizemos que uma $G$-ação é localmente suave (locally smooth) se existe um tubo linear sobre cada órbita do espaço. Neste caso, o conjunto dos pontos fixos por $G, M^{G}$, é uma subvariedade topológica de $M$. Observemos que esta definição, apesar de utilizar a nomenclatura "localmente suave", não se refere a aplicações e espaços diferenciáveis. Para as demonstrações e uma discussão em detalhes dos resultados, referimos o leitor aos livros $[\mathrm{Br}]$ e $[\mathrm{tD}]$.

Um G-par (metrizável) é um par $(M, B)$, onde $M$ é um $G$-espaço (metrizável) e $B$ é um $G$-subespaço fechado de $M$.

Sejam $(M, B)$ um $G$-par e $k: B \rightarrow M$ uma $G$-aplicação. Uma $G$-aplicação $\bar{k}: U \rightarrow$ $M$ é uma G-NE ( $G$-neighbourhood extension) de $k$ se $U$ é uma vizinhança invariante de $B$ e $\bar{k} \mid B=k$.

Um $G$-espaço $M$ é um G-ANE ( $G$-absolute neighbourhood extensor) se, para todo $G$-par metrizável $(X, A)$ e toda $G$-aplicação $k: A \rightarrow M$, existe uma $G$-NE $\bar{k}: U \rightarrow M$ de $k$.

Seja $(M, B)$ um $G$-par. O $G$-espaço $B$ é um G-NR ( $G$-neighbourhood retract) de $M$ se existe uma $G$-retração $r: U \rightarrow B$ de uma vizinhança invariante $U$ de $B$ em $M$.

Um $G$-espaço $B$ é um G-ANR ( $G$-absolute neighbourhood retract) se $B$ é metrizável e, se $B$ é um $G$-subespaço fechado de um $G$-espaço metrizável $M$, então $B$ é um $G$-NR de $M$.

Veremos agora alguns resultados, apresentados em [A] e em [M], que serão utilizados na demonstração da proposição (3.2.5). O teorema (3.1.1) é provado em ([M], Teorema 
8.8, p.501) e a teorema (3.1.2) pode ser encontrado em ([A], Teorema 14, p.530).

Teorema 3.1.1. Toda G-variedade localmente suave é um G-ANR.

Teorema 3.1.2. Sejam $G$ um grupo compacto e $M$ um G-espaço metrizável. Então $M$ é um $G$-ANR se, e somente se, $M$ é um $G$-ANE.

O resultado a seguir é o lema de Urysohn equivariante. Vide ([M], Lema 6.12, p.496)

Lema 3.1.3. Se $A$ e $B$ são $G$-subconjuntos fechados disjuntos de um G-espaço normal $M$, então existe uma aplicação equivariante $f: M \rightarrow I$ tal que $f(A)=0$ e $f(B)=1$.

\subsection{Propriedade Wecken Equivariante}

Iniciaremos a apresentação dos principais resultados deste trabalho supondo algumas hipóteses sobre o espaço $Y$.

Proposição 3.2.1. Sejam $G$ um grupo finito e $f: X \rightarrow Y$ uma $G$-aplicação entre dois espaços conexos por caminhos. Suponha $X$ compacto e $Y$ uma n-variedade topológica. Seja $a \in Y^{G} \neq \emptyset$ e suponha $Y$ simplesmente conexo. Se $N_{G}(f, a)=0$, então $f$ é G-homotópica a uma G-aplicação livre de raízes.

DemonstraÇÃo. Seja $\eta: \widehat{Y} \rightarrow Y$ o revestimento correspondo ao subgrupo $f_{\#} \pi_{1}(X)$ em $\pi_{1}(Y)$. Sendo $Y$ simplesmente conexo, então todo espaço de revestimento sobre $Y$ é equivalente ao espaço de revestimento trivial $i d: Y \rightarrow Y$. Portanto, $\eta$ é um homeomorfismo. Além disso, existe uma única $G$-classe de raízes $\mathcal{R}=f^{-1}(a)$ de $f$. Se $N_{G}(f, a)=0$, então $\mathcal{R}$ é inessencial. Logo, $f$ é $G$-homotópica a uma $G$-aplicação $f^{\prime}$ tal que $\mathcal{R}$ não está relacionada a nenhuma raiz de $f^{\prime}$. Seja $\hat{a} \in \eta^{-1}(a)$. Então existe um levantamento $\hat{f}^{\prime}: X \rightarrow \widehat{Y}$ de $f^{\prime}$ através de $\eta$ tal que $\hat{a} \notin \hat{f}^{\prime}(X)$, ou seja, $\hat{f}^{-1}(\hat{a})=\emptyset$. Deste modo, concluímos que $f^{\prime-1}(a)=\emptyset$. 
Lema 3.2.2. Suponha $\pi_{1}(Y)<\infty$ e suponha que o espaço de revestimento de Hopf de $Y$ seja o próprio $Y$. Então se $N_{G}(f, a)=0$, existe uma $G$-aplicação livre de raizes que é G-homotópica a f.

Demonstração. Sabemos, da teoria de espaços de revestimentos, que $\pi_{1}(Y)$ atua transitivamente na fibra $\eta^{-1}(a)$, onde $\eta$ é o revestimento de Hopf para $f$. Então $\eta^{-1}(a) \cong \pi_{1}(Y) / \eta_{\#} \pi_{1}(Y)$. Mas pelo teorema do isomorfismo, $\pi_{1}(Y) \cong \eta_{\#} \pi_{1}(Y)$, ou seja, $\eta$ é um homeomorfismo. Logo, utilizando a parte final da demonstração da proposição (3.2.1), o resultado segue.

Nos próximos resultados sobre a realização do $G$-número de Nielsen, quando este é zero, vamos assumir algumas condições sobre a $G$-ação no espaço de revestimento $\widehat{Y}$.

Suponha que a $G$-ação em $X, Y$ e em $\widehat{Y}$ seja localmente suave.

Teorema 3.2.3. Sejam $G$ um grupo finito, $X$ um $G$-espaço compacto e conexo por caminhos, $Y$ uma G-variedade topológica conexa por caminhos com dim $\widehat{Y}=n \geq 3$. Sejam $f: X \rightarrow Y$ uma aplicação equivariante e $a \in Y^{G} \neq \emptyset$. Suponha que $G$ atue livremente no espaço de revestimento de Hopf $\widehat{Y}$ de $Y$. Se $N_{G}(f, a)=0$, então existe uma $G$-aplicação livre de raízes $k: X \rightarrow Y$ G-homotópica a $f$.

Demonstração. Suponha que $f^{-1}(a)$ é não-vazio. Como $N_{G}(f, a)=0$, então todas as $G$-classes de raízes são inessenciais. Seja $\mathcal{R}$ uma $G$-classe de raízes inessencial de $f$ em $a$. Logo, $f$ é $G$-homotópica a uma $G$-aplicação $f^{\prime}$ tal que $\mathcal{R}$ não está relacionada a nenhuma raiz de $f^{\prime}$. Seja $\hat{a} \in \eta^{-1}(a)$. Então existe um levantamento $\hat{f}^{\prime}: X \rightarrow \widehat{Y}$ de $f^{\prime}$, através do revestimento de Hopf $\eta: \widehat{Y} \rightarrow Y$ para $f$, tal que $\hat{a} \notin \hat{f}^{\prime}(X)$. Logo, $G(\hat{a}) \cap \hat{f}^{\prime}(X)=\emptyset$.

Como $\hat{f}^{\prime}(X)$ é compacto, existe um número finito de pontos $\hat{y}_{1}, \ldots, \hat{y}_{m}$ em $\eta^{-1}(a) \cap$ $\hat{f}^{\prime}(X)$. Para todo $g \in G$, temos que $g \cdot \hat{y}_{j} \in \eta^{-1}(a) \cap \hat{f}^{\prime}(X), 1 \leq j \leq m$, pois $\eta$ e $\hat{f}^{\prime}$ são $G$-aplicações.

Considere as $G$-órbitas dos pontos $g \cdot \hat{y}_{j}$, para todo $g \in G$ e $j=1, \ldots, m$. Escolha um representante para cada uma destas órbitas. Denote tais $G$-órbitas por $G\left(z_{1}\right), \ldots, G\left(z_{l}\right)$, 
onde $z_{i}$ é um representante escolhido de $G\left(z_{i}\right), i=1, \ldots, l$. Observe que como $G$ é um grupo finito agindo livremente em $\widehat{Y}$, então $\# G(\hat{y})=\# G$, para todo $\hat{y} \in \widehat{Y}$. Além disso, a aplicação de órbita $p: \widehat{Y} \rightarrow \widehat{Y} / G$ é uma aplicação de revestimento.

Seja $c: I \rightarrow \widehat{Y} / G$ um caminho simples, isto é, sem auto-interseção, de $p(\hat{a})=G(\hat{a})$ até $G\left(z_{l}\right)$ passando apenas uma vez pelas órbitas $G\left(z_{1}\right), \ldots, G\left(z_{l-1}\right)$. Logo, existe um único caminho $\hat{c}: I \rightarrow \widehat{Y}$ levantamento de $c$ por $p$, isto é, $p \hat{c}=c$ tal que $\hat{c}(0)=$ $\hat{a}$. Além disso, $\hat{c}$ é um caminho simples que passa apenas uma vez por cada órbita $G(\hat{a}), G\left(z_{1}\right), \ldots, G\left(z_{l-1}\right), G\left(z_{l}\right)$ com ponto final $\hat{c}(1)=g \cdot z_{l}$, para algum $g \in G$ e tal que $\hat{c}(I) \cap g \cdot \hat{c}(I)=\emptyset$, para todo $g \neq e$ onde $e$ denota o elemento identidade de $G$.

Seja $V_{c}$ uma vizinhança fechada de $c$ homeomorfa a uma $n$-bola fechada. Então $V_{c}$ é contrátil e $p^{-1}\left(V_{c}\right)=\left\{g \cdot V_{\hat{c}} \mid g \in G\right\}$, onde $V_{\hat{c}}$ é a vizinhança fechada correspondente de $\hat{c}$. Logo, $p^{-1}\left(V_{c}\right)$ consiste de transladados disjuntos de $V_{\hat{c}}$ por $G$. Observe que para cada $g \in G, g \cdot V_{\hat{c}}$ é uma vizinhança fechada do caminho $g \cdot \hat{c}$. Agora, use $g \cdot \hat{a}$ como o centro de cada bola $g \cdot V_{\hat{c}}$.

Considere $r: V_{\hat{c}}-\{\hat{a}\} \rightarrow \partial V_{\hat{c}}$ a projeção radial. Logo, $V_{\hat{c}}-\{\hat{a}\}$ é um retrato forte por deformação para sua fronteira $\partial V_{\hat{c}}$. Agora, para cada $g \in G$, defina $r_{g}$ : $g \cdot V_{\hat{c}}-\{g \cdot \hat{a}\} \rightarrow \partial\left(g \cdot V_{\hat{c}}\right)$ por $r_{g}(\hat{w})=g \cdot\left(r\left(g^{-1} \cdot \hat{w}\right)\right)$. Note que $r_{e}=r$. Finalmente, defina a $G$-retração:

$$
\bar{r}: \bigcup_{g \in G} g \cdot V_{\hat{c}}-G(\hat{a}) \rightarrow \bigcup_{g \in G} \partial\left(g \cdot V_{\hat{c}}\right)
$$

por $\bar{r}(\hat{y}):=r_{g}(\hat{y})$ para $\hat{y} \in g \cdot V_{\hat{c}}$. Então $\bigcup_{g \in G} g \cdot V_{\hat{c}}-G(\hat{a})$ é um $G$-retrato forte por deformação para sua fronteira $\bigcup_{g \in G} \partial\left(g \cdot V_{\hat{c}}\right)$. Seja $\zeta: \widehat{Y}-G(\hat{a}) \rightarrow \widehat{Y}$ uma $G$-aplicação dada por $\zeta(\hat{y})=\bar{r}(\hat{y})$ se $\hat{y} \in \bigcup_{g \in G} g \cdot V_{\hat{c}}-G(\hat{a})$ e $\zeta(\hat{y})=\hat{y}$ caso contrário.

Como $G(\hat{a}) \cap \hat{f}^{\prime}(X)=\emptyset$, pode-se compor $\hat{f}^{\prime} \operatorname{com} \zeta$ e assim, $\zeta \hat{f}^{\prime}(X) \cap \eta^{-1}(a)=\emptyset$. Portanto, a $G$-aplicação $\eta \zeta \hat{f}^{\prime}$ não tem raízes em $a$ e, além disso, $\eta \zeta \hat{f}^{\prime}$ é $G$-homotópica a $f^{\prime}$, que por sua vez é $G$-homotópica a $f$. Então $\eta \zeta \hat{f}^{\prime}$ é a $G$-aplicação desejada.

Proposição 3.2.4. Sejam $G$ um grupo finito, $f: X \rightarrow Y$ uma aplicação equivariante entre $G$-espaços conexos por caminhos e $a \in Y^{G} \neq \emptyset$. Suponha $X$ compacto e $Y$ uma 
G-variedade topológica. Suponha também que $G$ atue no espaço de revestimento de Hopf $\widehat{Y}$ de $Y$ com um único tipo de órbita $(H)$, onde $H$ é um subgrupo fechado de $G$ com $W H=N H / H$ finito e tal que $\widehat{Y}^{H}$ é conexo por caminhos com $\operatorname{dim} \widehat{Y}^{H} \geq 3$. Se $N_{G}(f, a)=0$ então $f$ é G-homotópica a uma $G$-aplicação livre de raízes.

Demonstração. Como $G$ atua em $\widehat{Y}$ com um único tipo de órbita $(H)$, então pelo lema (1.2.1) seque que $\widehat{Y}_{H}=\widehat{Y}^{H}$. Além disso, cada $G$-órbita em $\widehat{Y}$ contém um ponto fixado por $H$. Logo, para cada $\hat{y} \in \widehat{Y}$ existe $g \in G$ tal que $G_{g \cdot \hat{y}}=H$, onde $G_{g \cdot \hat{y}}$ denota o grupo de isotropia do elemento $g \cdot \hat{y}$. Observe ainda que $W H$ é finito e age livremente em $\widehat{Y}_{H}$, onde a $W H$-ação é induzida pela $G$-ação em $\widehat{Y}$, ou seja, $n H * \hat{y}=n \cdot \hat{y}:=\varphi(n)(\hat{y})$, para todo $n \in N H \subset G$ e para todo $\hat{y} \in \widehat{Y}^{H}$.

Suponha que $f^{-1}(a)$ seja não-vazio. Como $N_{G}(f, a)=0$, então todas as $G$-classes de raízes são inessenciais. Seja $\mathcal{R}$ uma $G$-classe de raízes inessencial de $f$ em $a$. Logo, $f$ é $G$-homotópica a uma $G$-aplicação $f^{\prime}$ tal que $\mathcal{R}$ não está relacionada a nenhuma raiz de $f^{\prime}$. Seja $\hat{a} \in \eta^{-1}(a)$. Então existe um levantamento $\hat{f}^{\prime}: X \rightarrow \widehat{Y}$ de $f^{\prime}$, através do revestimento de Hopf $\eta: \widehat{Y} \rightarrow Y$ para $f$, tal que $\eta \hat{f}^{\prime}=f^{\prime}$ e $G(\hat{a}) \cap \hat{f}^{\prime}(X)=\emptyset$. Sem perda de generalidade, suponha que $G_{\hat{a}}=H$.

Então, pelo teorema (3.2.3), para cada $n H \in W H$ obtem-se a retração $r_{n H}: n H *$ $V_{\hat{c}}-\{n H * \hat{a}\} \rightarrow \partial\left(n H * V_{\hat{c}}\right)$ dada por $r_{n H}(\hat{w})=n H *\left(r\left(n^{-1} H * \hat{w}\right)\right)=n \cdot\left(r\left(n^{-1} \cdot \hat{w}\right)\right)$, onde $r: V_{\hat{c}}-\{\hat{a}\} \rightarrow \partial V_{\hat{c}}$ é projeção radial. As vizinhanças fechadas $V_{\hat{c}}$, homeomorfas a $n$-bolas, em $\widehat{Y}^{H}$ foram obtidas como na demostração do teorema (3.2.3).

Logo, a $W H$-retração

$$
\bar{r}: \bigcup_{n H \in W H} n H * V_{\hat{c}}-W H(\hat{a}) \rightarrow \bigcup_{n H \in W H} \partial\left(n H * V_{\hat{c}}\right)
$$

dada por $\bar{r}(\hat{y})=r_{n H}(\hat{y})$ para $\hat{y} \in n H * V_{\hat{c}}$, garante que $\bigcup_{n H \in W H} n H * V_{\hat{c}}-W H(\hat{a})$ é uma $W H$-retração forte por deformação para sua fronteira $\bigcup_{n H \in W H} \partial\left(n H * V_{\hat{c}}\right)$. Então considere a $W H$-homotopia $F:\left(\bigcup_{n H \in W H} n H * V_{\hat{c}}-W H(\hat{a})\right) \times I \rightarrow \bigcup_{n H \in W H} n H * V_{\hat{c}}-$ $W H(\hat{a})$ tal que $F(\hat{y}, 0)=\hat{y}, F(\hat{y}, 1)=\bar{r}(\hat{y})$ para todo $\hat{y} \in \bigcup_{n H \in W H} n H * V_{\hat{c}}-W H(\hat{a})$ e $F(\hat{w}, t)=\hat{w}$ para todo $\hat{w} \in \bigcup_{n H \in W H} \partial\left(n H * V_{\hat{c}}\right)$ e $t \in I$. Pelo corolário (1.2.6), $F$ tem 
uma extensão para uma $G$-aplicação $F^{\prime}:\left(\bigcup_{g \in G} g * V_{\hat{c}}-G(\hat{a})\right) \times I \rightarrow \bigcup_{g \in G} g * V_{\hat{c}}-G(\hat{a})$, onde $F^{\prime}(g \cdot \hat{y}, t)=g \cdot F(\hat{y}, t), G_{\hat{y}}=g H g^{-1}$ para todo $\hat{y} \in \widehat{Y}^{H}$.

Seja $\bar{F}: \widehat{Y}-G(\hat{a}) \times I \rightarrow \widehat{Y}$ a $G$-aplicação dada por $\bar{F}(\hat{y}, t)=F^{\prime}(\hat{y}, t)$ se $\hat{y} \in$ $\bigcup_{g \in G} g * V_{\hat{c}}-G(\hat{a})$ e $\bar{F}(\hat{y}, t)=\hat{y}$ caso contrário.

Denote $\bar{F}(\hat{w}, 1)$ por $\bar{F}_{1}(\hat{w})$, para todo $\hat{w} \in \widehat{Y}-G(\hat{a})$. Logo, como $G(\hat{a}) \cap \hat{f}^{\prime}(X)=\emptyset$, pode-se compor as $G$-aplicações $\hat{f}^{\prime}$ e $\bar{F}_{1}$ e obter $\bar{F}_{1} \hat{f}^{\prime}(X) \cap \eta^{-1}(a)=\emptyset$. Portanto, a $G$-aplicação $\eta F_{1}^{\prime} \hat{f}^{\prime}$ não tem raízes em $a$ e é $G$-homotópica a $f^{\prime}$, que por sua vez, é $G$-homotópica a $f$.

Proposição 3.2.5. Sejam $G$ um grupo finito, $f: X \rightarrow Y$ uma aplicação equivariante entre $G$-espaços conexos por caminhos e a $\in Y^{G} \neq \emptyset$. Suponha $X$ compacto e $Y$ uma $G$-variedade topológica. Suponha também que $G$ atue semi-livremente no espaço de revestimento de Hopf $\widehat{Y}$ de $Y$. Além disso, suponha que $\widehat{Y}$ e $\widehat{Y}^{G}$ sejam conexos por caminhos, $\operatorname{dim} \widehat{Y}^{G}=n \geq 3$ e que a codimensão de $\widehat{Y}^{G}$ em $\widehat{Y}$ seja pelo menos 2 . Então, se $N_{G}(f, a)=0$, existe uma $G$-aplicação livre de raízes $G$-homotópica a $f$.

Demonstração. Primeiramente, note que, como a $G$-ação em $\widehat{Y}$ é semi-livre, então $\widehat{Y}=\widehat{Y}^{G} \cup \widehat{Y}_{e}$. Suponha que $f^{-1}(a)$ seja não-vazio. Seja $\eta: \widehat{Y} \rightarrow Y$ o revestimento de Hopf para $f$ e seja $\hat{f}: X \rightarrow \widehat{Y}$ um levantamento de $f$ através de $\eta$.

Temos dois casos a considerar.

Caso I. $\hat{f}(X) \cap \widehat{Y}^{G}=\emptyset$.

Como o conjunto $\hat{f}(X) \neq \emptyset$ é fechado em $\widehat{Y}$, que é conexo, então existe um ponto $\hat{b} \in \widehat{Y}_{e}$ tal que $\hat{b} \notin \hat{f}(X)$. Portanto, pelo teorema (3.2.3), o resultado segue.

Caso II: $\hat{f}(X) \cap \hat{Y}^{G} \neq \emptyset$.

Como $N_{G}(f, a)=0$, então todas as $G$-classes de raízes são inessenciais. Seja $\mathcal{R}$ uma $G$-classe de raízes inessencial de $f$. Logo, $f$ é $G$-homotópica a uma $G$-aplicação $f^{\prime}$ tal que $\mathcal{R}$ não está relacionada a nenhuma raiz de $f^{\prime}$. Seja $\hat{f}^{\prime}: X \rightarrow \widehat{Y}$ um levantamento de Hopf de $f^{\prime}$ por $\eta$.

Para o caso II, vamos precisar da seguinte hipótese adicional: seja $\hat{a}$ um ponto 
em $\widehat{Y}$ tal que $\hat{a} \in \widehat{Y}^{G} \cap \eta^{-1}(a)$ e $\hat{a} \notin \hat{f}^{\prime}(X)$.

Como $\hat{f}^{\prime}(X)$ é compacto, existe um número finito de pontos $\hat{y}_{1}, \ldots, \hat{y}_{m}$ em $\eta^{-1}(a) \cap$ $\hat{f}^{\prime}(X)$. Para todo $g \in G$, temos que $g \cdot \hat{y}_{j} \in \eta^{-1}(a) \cap \hat{f}^{\prime}(X), 1 \leq j \leq m$, pois $\eta$ e $\hat{f}^{\prime}$ são $G$-aplicações.

Considere as $G$-órbitas dos pontos $g \cdot \hat{y}_{j}$, para todo $g \in G$ e $j=1, \ldots, m$. Escolha um representante para cada uma destas órbitas. Denote tais $G$-órbitas por $G\left(z_{1}\right), \ldots, G\left(z_{l}\right)$, onde $z_{i}$ é um representante escolhido de $G\left(z_{i}\right), i=1, \ldots, l$. Como $G$ atua semi-livremente em $\widehat{Y}$, então os pontos em $\eta^{-1}(a) \cap \hat{f}^{\prime}(X)$ são particionados em dois conjuntos disjuntos, a saber, aqueles que pertencem a parte fixada por $G, \widehat{Y}^{G}$, e aqueles que pertecem a parte livre $\widehat{Y}-\widehat{Y}^{G}=\widehat{Y}_{e}$.

Sejam $z_{1}, z_{2}, \ldots, z_{s} \in \hat{f}^{\prime}(X) \cap \eta^{-1}(a) \cap \widehat{Y}^{G}$, para algum $s$ tal que $1 \leq s \leq l$. Como $\widehat{Y}^{G}$ é uma subvariedade fechada de $\widehat{Y}$, obtemos (pelo Lema 1 de [DH]) uma vizinhança fechada $D$, homeomorfa a uma $n$-bola fechada, contida em $\hat{Y}^{G}$ contendo os pontos $\hat{a}$, $z_{1}, z_{2}, \ldots, z_{s}$. Agora, considerando $\hat{a}$ como o centro de $D$, obtemos a projeção radial $r: D-\{\hat{a}\} \rightarrow \partial D$. Logo, $\partial D$ é um retrato forte por deformação de $D-\{\hat{a}\}$, ou seja, existe uma homotopia $F:(D-\{\hat{a}\}) \times I \rightarrow D$ tal que $F(\hat{y}, 0)=\hat{y}, F(\hat{y}, 1)=r(\hat{y})$ para todo $\hat{y} \in D-\{\hat{a}\}$ e $F(\hat{w}, t)=\hat{w}$ para todo $\hat{w} \in \partial D, t \in I$. Agora, seja $F^{\prime}:\left(\widehat{Y}^{G}-\{\hat{a}\}\right) \times I \rightarrow \widehat{Y}^{G}$ dada por $F^{\prime}(\hat{y}, t)=F(\hat{y}, t)$ se $\hat{y} \in D-\{\hat{a}\}$ e $F^{\prime}(\hat{y}, t)=\hat{y}$ caso contrário.

Pelo teorema (3.1.1), $\widehat{Y}^{G}$ é um $G$-ANR. Então $\widehat{Y}^{G}$ é um $G$-ANE por (3.1.2). Portanto, existe uma $G$-vizinhança $U$ de $\widehat{Y}^{G}$ em $\widehat{Y}$ e existe uma $G$-aplicação $\bar{F}: U \times I \rightarrow \widehat{Y}$ tal que $\bar{F} \mid\left(\widehat{Y}^{G} \times I\right)=F^{\prime}$.

Considere os subconjuntos fechados invariantes disjuntos $\widehat{Y}-U$ e $\widehat{Y}^{G}$ do $G$-espaço normal $\widehat{Y}$, então pelo lema de Urysohn equivariante, existe uma $G$-aplicação $\psi: \widehat{Y} \rightarrow I$ tal que $\psi(\widehat{Y}-U)=0$ e $\psi\left(\widehat{Y}^{G}\right)=1$.

Agora, defina (pois $\widehat{Y}$ é metrizável) a $G$-aplicação $\overline{\bar{F}}: \widehat{Y} \times I \rightarrow \widehat{Y}$ por $\overline{\bar{F}}(\hat{y}, t)=$ $\psi(\hat{y}) \bar{F}(\hat{y}, t)+(1-\psi(\hat{y})) \hat{y}$. Logo, $\overline{\bar{F}}(\hat{y}, t)=\bar{F}(\hat{y}, t)$, para todo $\hat{y} \in \widehat{Y}^{G}$ e $\overline{\bar{F}}(\hat{y}, t)=\hat{y}$, para $\hat{y} \in \widehat{Y}-U$. Portanto, $\overline{\bar{F}} \mid U=\bar{F}$ e $\overline{\bar{F}} \mid \widehat{Y}^{G}=F^{\prime}$. 
Denote $\overline{\bar{F}}(\hat{y}, 1)$ por $\overline{\bar{F}}_{1}(\hat{y})$, para todo $\hat{y} \in \widehat{Y}$ e, seja $\xi=\overline{\bar{F}}_{1} \hat{f}^{\prime}$. Então, $\xi(X) \cap \eta^{-1}(a) \subset$ $\widehat{Y}_{e}$.

Seja $\xi(X) \cap \eta^{-1}(a)=\left\{G\left(z_{s+1}\right), \ldots, G\left(z_{l}\right)\right\} \subset \widehat{Y}_{e}$. Seja $c: I \rightarrow \widehat{Y} / G$ um caminho simples no espaço de órbitas $\widehat{Y} / G$ de $p(\hat{a})=\hat{a}$ para $G\left(z_{k}\right)$, para algum $k \in\{s+1, \ldots, l\}$. Então existe um levantamento $\hat{c}: I \rightarrow \widehat{Y}$ de $c$ por $p: \widehat{Y} \rightarrow \widehat{Y} / G$, isto é $p \hat{c}=c$, tal que $\hat{c}(0)=\hat{a}$ e $\hat{c}(1)=g^{\prime} \cdot z_{k}$, para algum $g^{\prime} \in G$. Observe que, como a codimensão de $\widehat{Y}^{G}$ em $\widehat{Y}$ é pelo menos 2, o caminho $\hat{c}$ pode ser tomado como um caminho simples interceptando $\widehat{Y}^{G}$ em um único ponto, a saber $\hat{a}$. Além disso, podemos assumir que $\hat{c}$ evita todos os outros pontos de $G\left(z_{s+1}\right), \ldots, G\left(z_{l}\right)$, exceto $g^{\prime} \cdot z_{k}$.

Portanto, os caminhos simples $g \hat{c}: I \rightarrow \widehat{Y}$ começam em $\hat{a}$ e terminam em um ponto da órbita $G\left(z_{k}\right)$ e são tais que $g \hat{c}(I) \cap \hat{c}(I)=\hat{a}$, para todo $g \in G, g \neq e$. Como $\widehat{Y}_{e}$ é normal e os caminhos $g \hat{c}(I)$ - $\hat{a}$ são fechados em $\widehat{Y}_{e}$, então eles são separados por vizinhanças abertas disjuntas. Denote por $V_{g \hat{c}}^{\prime}$ tal vizinhança de $g \hat{c}(I)-g \hat{a}$ em $\widehat{Y}_{e}$, para cada $g \in G$. Seja $V_{\hat{c}}$ uma vizinhança fechada (homeomorfa a uma bola fechada) de $\hat{c}$ em $\widehat{Y}$ tal que o conjunto $V_{\hat{c}}-\{\hat{a}\}$ está contido no interior de $V_{\hat{c}}^{\prime}$. Então, $\hat{a} \in \partial V_{\hat{c}}, \hat{c}(1)=g^{\prime} \cdot z_{k}$ pertence ao interior de $V_{\hat{c}}, V_{\hat{c}} \cap \widehat{Y}^{G}=\hat{a}$ (isto é, $V_{\hat{c}}-\{\hat{a}\} \subset \widehat{Y}_{e}$ ) e $V_{\hat{c}} \cap g \hat{c}(I)=\hat{a}$, para todo $g \neq e$ em $G$. Logo, $p^{-1}\left(p\left(V_{\hat{c}}\right)\right)=\bigcup_{g \in G} g \cdot V_{\hat{c}}$ e $\bigcap_{g \in G} g \cdot V_{\hat{c}}=\{\hat{a}\}$.

Considere a projeção radial $\rho: V_{\hat{c}}-\{\hat{a}\} \rightarrow V_{\hat{c}}$, onde todos os raios começam no ponto $\hat{a}$. Então $\rho\left(g^{\prime} \cdot z_{k}\right) \in \partial V_{\hat{c}}$. Logo, para cada $g \in G$, defina $\rho_{g}: g \cdot V_{\hat{c}}-\{\hat{a}\} \rightarrow g \cdot V_{\hat{c}}$ por $\rho_{g}(\hat{y})=g \cdot\left(\rho\left(g^{-1} \cdot \hat{y}\right)\right)$. Seja, agora,

$$
\bar{\rho}: \bigcup_{g \in G} g \cdot V_{\hat{c}}-\{\hat{a}\} \longrightarrow \bigcup_{g \in G} g \cdot V_{\hat{c}}
$$

a $G$-aplicação dada por $\bar{\rho}(\hat{y})=\rho_{g}(\hat{y})$ para $\hat{y} \in g \cdot V_{\hat{c}}$. Finalmente, defina a $G$-aplicação $\zeta: \widehat{Y}-\{\hat{a}\} \rightarrow \widehat{Y}$ por $\zeta(\hat{y})=\bar{\rho}(\hat{y})$ se $\hat{y} \in \bigcup_{g \in G} g \cdot V_{\hat{c}}-\{\hat{a}\}$ e $\zeta(\hat{y})=\hat{y}$ caso contrário. Portanto $\zeta \hat{f}^{\prime}(X) \cap \eta^{-1}(a)=\left\{G\left(z_{s+1}\right), \ldots, G\left(z_{k-1}\right), G\left(z_{k+1}\right), \ldots, G\left(z_{l}\right)\right\} \subset \widehat{Y}_{e}$, onde $s+1 \leq$ $k \leq l$. Repetindo este processo, um número finito de vezes, para eliminar as $G$-órbitas restantes (que produzem raízes), obtemos uma $G$-aplicação $\sigma: \widehat{Y}-\{\hat{a}\} \rightarrow \widehat{Y}$ tal que $\eta \sigma \hat{f}^{\prime}: X \rightarrow Y$ é $G$-homotópica a $f$ e é livre de raízes. 


\section{Considerações finais}

Em [B1], R. Brooks mostrou que se $Y$ é uma variedade topológica então $N(f, a)=0$ ou $N(f, a)=R(f, a)$. Ou seja, se uma classe de raízes é essencial então todas as classes de raízes são essenciais. Isto nos motiva a perguntar: sob que condições, se uma $G$-classe de raízes é essencial então todas são? Para responder a essa pergunta, consideremos uma série de resultados, os quais são conseqüências importantes do teorema (3.2.3) e das proposições $(3.2 .4)$ e (3.2.5).

Nos lemas a seguir, suponha que a $G$-ação em $X, Y$ e em $\widehat{Y}$ seja localmente suave.

Lema 3.2.6. Sejam $G$ um grupo finito, $X$ um $G$-espaço compacto e conexo por caminhos, $Y$ uma G-variedade topológica conexa por caminhos com $\operatorname{dim} \widehat{Y}=n \geq 3$. Sejam $f: X \rightarrow Y$ uma aplicação equivariante e $a \in Y^{G} \neq \emptyset$. Suponha que $G$ atue livremente no espaço de revestimento de Hopf $\widehat{Y}$ de $Y$. Se f possui uma G-classe de raízes inessencial, então existe uma G-aplicação livre de raizes G-homotópica a f.

DemonstraçÃo. Segue imediatamente da demonstração do teorema (3.2.3).

Lema 3.2.7. Sejam $G$ um grupo finito, $f: X \rightarrow Y$ uma aplicação equivariante entre $G$-espaços conexos por caminhos e $a \in Y^{G} \neq \emptyset$. Suponha $X$ compacto e $Y$ uma $G$ variedade topológica. Suponha também que $G$ atue no espaço de revestimento de Hopf $\widehat{Y}$ de $Y$ com um único tipo de órbita $(H)$, onde $H$ é um subgrupo fechado de $G$ com $W H=N H / H$ finito e tal que $\widehat{Y}^{H}$ é conexo por caminhos com $\operatorname{dim} \widehat{Y}^{H} \geq 3$. Se $f$ possui uma $G$-classe de raúzes inessencial, então existe uma $G$-aplicação livre de raúzes G-homotópica a $f$. 
Demonstração. Segue imediatamente da demonstração da proposição (3.2.4).

Lema 3.2.8. Sejam $G$ um grupo finito, $f: X \rightarrow Y$ uma aplicação equivariante entre $G$-espaços conexos por caminhos e a $\in Y^{G} \neq \emptyset$. Suponha $X$ compacto e $Y$ uma G-variedade topológica. Suponha também que $G$ atue semi-livremente no espaço de revestimento de Hopf $\widehat{Y}$ de $Y$. Além disso, suponha que $\widehat{Y}$ e $\widehat{Y}^{G}$ sejam conexos por caminhos, $\operatorname{dim} \widehat{Y}^{G}=n \geq 3$ e que a codimensão de $\widehat{Y}^{G}$ em $\widehat{Y}$ seja pelo menos 2. Se $f$ possui uma $G$-classe de raízes inessencial, então existe uma G-aplicação livre de raízes G-homotópica a $f$.

DemonstraÇÃo. Segue imediatamente da demonstração da proposição (3.2.5).

Portanto, concluímos, de cada lema acima, que se $f$ tem uma $G$-classe de raízes essencial, então todas as $G$-classes de raízes $f$ são essenciais. Ou seja, $N_{G}(f, a)=0$ ou $N_{G}(f, a)=R_{G}(f, a)$, nos casos acima.

Nosso próximo objetivo é provar uma versão mais geral da proposição (3.2.5), ou seja, estudar o caso em que $G$ age em $\widehat{Y}$ com um número finito de tipos de isotropias $\left(H_{1}\right),\left(H_{2}\right), \ldots,\left(H_{k}\right)$. A dificuldade, como pode ser observada na demostração da proposição (3.2.5), vem do fato de que os pontos que contribuem para as raízes podem ter tipos de isotropias diferentes. 


\section{Referências Bibliográficas}

[A] S. Antonian, Equivariant embeddings into G-AR's. Glasnik Math. Ser. III 22 (42) (1987), 503-533.

[BCW] L. Borsari, F. Cardona and P. Wong, Equivariant Path Fields on Topological Manifolds, to appear in T.M.N.A.

[Br] G. Bredon, Introduction to Compact Transformation Groups. Academic Press, New York, 1992.

[B] R. Brooks, Coincidences, Roots and Fixed Points. Ph.D. thesis, University of California at Los Angeles, Los Angeles, 1967.

[B1] R. Brooks, Certain subgroups of the fundamental group and the number of roots of $f(x)=a$. Amer. J. Math. 95 (1973), 720-728.

[B2] R. Brooks, On the sharpness of the $\Delta_{1}$ and $\Delta_{2}$ Nielsen numbers. J. Reine Angew. Math. 259 (1973), 101-108.

[B3] R. Brooks, Nielsen root theory. Handbook of Topological Fixed Point Theory, Springer-Verlag, New York (2006), 375-431.

[BS] R. Brown and H Schirmer, Nielsen root theory and Hopf degree theory. Pacific Journal of Mathematics, 198 (2001), 49-80.

[DH] P. Doyle and J. Hocking, A decomposition theorem for n-dimensional manifolds. Proc. Amer. Math. Soc. 13 (1962), 469-471. 
[Fa] P. Fagundes, Teoria de Coincidência Equivariante e Números de Nielsen Equivariantes. Tese de Doutorado, USP-São Paulo, 1996.

[GH] J. Guo and P. Heath, Equivariant coincidence Nielsen numbers. Topology Appl. 128 (2003), 277-308.

[GW] D. Gonçalves and P. Wong, Wecken property for roots. Proc. Amer. Math. Soc. 133 (2005), 2779-2782.

[H] H. Hopf, Zur Topologie der Abbildungen von Mannigfaltigkeiten, II. Math. Ann. 102 (1930), 562-623.

[Hu] Sze-Tsen Hu, Homotopy Theory. Academic Press, 1959.

[J] B. Jiang, Lectures on Nielsen Fixed Point Theory. Contemporary Mathematics 14, American Mathematical Society, Providence, 1983.

[Ka] K. Kawakubo, The Theory of Transformation Groups. Oxford University Press, 1991.

[K] T. Kiang, The Theory of Fixed Point Classes. Science Press, Springer, BerlinBeijing, 1989.

[M] M. Murayama, On G-ANR's and their G-homotopy types. Osaka J. Math. 20 (1983), 479-512.

[R] J. Rotman, An Introduction to Algebraic Topology. Springer-Verlag, New York, 1988 .

[S] H. Schirmer, Mindestzahlen von Koinzidenpunkten. J. Reine Angew. Math. 194 (1955), 21-39.

[tD] T. tom Dieck, Transformation groups, Studies in Mathematics 8, de Gruyter, Berlin, 1987. 
[V] J. Vick, Homology Theory. 2nd ed, Springer-Verlag, New York, 1994.

[We] F. Wecken, Fixpunktklassen. I, Math. Ann. 117 (1941), 659-671; II 118 (1942), 216-234; III 118 (1942), 544-577.

[W1] P. Wong, Equivariant Nielsen fixed point theory for G-maps. Pacific Journal of Mathematics, vol.150 n.120 (1991), 179-200.

[W2] P. Wong, Equivariant Nielsen numbers. Pacific Journal of Mathematics. 159 (1993), 153-175.

[W3] P. Wong, Fixed point theory for homogeneous spaces. Amer. J. Math. 120 (1998), 23-42.

[W4] P. Wong, Equivariant Nielsen theory. Nielsen theory and Reidemeister torsion. Banach Center Publications 49 (1999), 253-258.

[W5] P. Wong, Fixed point theory for homogeneous spaces II. Fund. Mathematicae 186 (2005), 161-175. 